\title{
Monetary Shocks in a Spatial Overlapping Generations Model
}

\author{
Niels Anthonisen \\ Department of Economics, Mount Allison University
}

September, 2007.

\begin{abstract}
The paper combines a simple monetary overlapping generations model with a simple abstract spatial structure, to produce a model that focuses on the effect of the distribution of cash balances on real and nominal variables, both across the economy, and over time. The model is then used to study the effects of a monetary shock.

The economy is endowed with a spatial structure in the form of a Cayley graph. Overlapping generations of two-period lived agents inhabit the vertices of the graph. Agents produce when young and consume when old. However, agents only consume the goods produced by their neighbours. All transactions are mediated by money. In her first period of life, a generic agent produces output and sells it to her old neighbours in exchange for cash; she then carries the newly acquired cash balances into her second period of life, and uses them to purchase consumption goods from her young neighbours. Therefore, money flows through the economy, traveling from one vertex to adjacent vertices as a consequence of production and exchange.

The distribution of money across the economy determines relative prices which, in turn, determines the profile of production across the economy. Therefore any monetary shock that alters the distribution of money will also have an impact on both the distribution and the level of production.
\end{abstract}




\section{Introduction}

The paper combines a simple monetary overlapping generations model with a simple spatial structure, in order to produce a model that focuses explicity on the effects of the distribution of cash balances on prices and production, both across the economy, and over time. The model is then used to study the effects of a monetary shock in such an economy.

The economy is endowed with a spatial structure in the form of a graph such as the one shown in Figure 1. Overlapping generations of two-period lived agents inhabit the vertices of the graph; specifically, at any moment in time two agents, one young and one old, are located at each vertex. Individuals are stationary in that each agent remains at the same vertex for both periods of her life. Agents produce when young and consume when old. However, agents only consume the goods produced by their neighbours, where two agents are neighbours if they inhabit adjacent vertices. (Two vertices are adjacent if they are connected by an edge.) All transactions are mediated by currency. At the outset of any time period, all cash balances in the economy are held by the old agents, where, as mentioned, one old agent inhabits each vertex. In her first period of life, a generic agent produces output and sells it to her old neighbours in exchange for cash; she then carries the newly acquired cash balances into her second period of life, where they are used to purchase consumption goods from her young neighbours. Therefore, at any moment in time, currency is distributed across the vertices of the graph. It then flows throughout the economy, traveling from one vertex to adjacent vertices as a consequence of production and exchange.

At the core of the paper is an experiment whereby a monetary shock occurs in period 1 in the form of unanticipated transfers to a subset of old agents. The main objective of the paper is to trace the effects of this shock as it diffuses through the economy. The fundamental fact of the model is that the distribution of currency across the economy determines relative prices which, in turn, determine production levels. Therefore, a monetary shock that alters the distribution of money will have an impact on output both across the economy, and over time.

Changes in money effect real economic activity because prices respond differentially to a monetary disturbance. Specifically, prices at locations that are close to the monetary disturbance change to a greater extent than do prices at locations that are more distant from the disturbance. Consequently, the model illustrates the important fact that sticky prices (or other frictions) are not necessary for money to have an effect on real economic activity. Instead, it is enough for different markets to have different linkages to the source of a monetary shock. Moreover, something like this must happen in real economies: Changes in monetary policy spread through the economy in a pattern that 
reflects the connections between different markets. The most immediate impact of a change in monetary policy is on markets for short-term debt; from there, the disturbance is transmitted to markets for longer-term debt, and then to other asset markets, and then ultimately to goods and labour markets.

In the model, if currency is distributed uniformly over the locations in the economy, then all agents produce identical quantities and sell their output at identical prices. Moreover, any change in the money stock that maintains a uniform distribution of currency, will have an immediate effect on the price level, without altering real economic variables whatsoever. In other words, changes in money are neutral as long as the distribution of cash balances remains uniform. Thus a change in the distribution of currency is the only factor that can cause a change in either aggregate output, or in the distribution of output across agents. Therefore, the model provides a convenient laboratory for examining the role of the distribution of money in influencing real economic activity.

One of the model's most attractive features is its tractability. Three assumptions account for this. The first is the assumption of overlapping generations, the second is that consumers' preferences over consumption goods are Cobb-Douglas, and the third is that the graphs in the paper possess a high degree of symmetry in that each vertex has the same local structure as every other vertex. (Equivalently, each neighbourhood appears identical in its spatial characteristics from the point of view of any individual agent.) More specifically, all graphs in the paper belong to the family of Cayley graphs; these comprise a reasonably broad class that are described and motivated in due course. However, a fact that is useful to know at the moment is that the vertices of a Cayley graph are identified with the elements of group (in the algebraic sense). Together these two features - Cobb-Douglas preferences, and homogeneous neighbourhood structure imply that the distribution of money evolves according to a time-invariant process that can be completely described by an $n \times n$ matrix $A$, where $n$ is the number of locations in the economy (equivalently, the number of vertices in the graph). This fact enables tremendous simplification. It means, among other things, that closed-form expressions can be obtained for nearly all economic variables of interest. Furthermore, the combination of Cobb-Douglas preferences with the assumption that the underlying graph is built from the elements of a group, further simplifies the analysis, for together these features imply that the diffusion of money through the economy is naturally compatible with the underlying group operation (this is described more completely in Subsection BLANK). Moreover, this compatibility renders the analysis of the diffusion of money amenable to investigation via powerful tools of group representation theory. Under certain additional assumptions, a complete eigenvalue/eigenvector decomposition of the matrix $A$ becomes possible. This greatly enhances the tractability of the model. For instance, some extremely large economies can be investigated through the methods employed in 
the paper. Indeed, perhaps the models' most appealing feature is that it occupies that slim intersection of two classes of models: those that are sufficiently complicated as to be interesting, and those that are sufficiently tractable as to admit a reasonably complete analysis.

The paper's primary objective is to investigate the effect of a monetary shock on real variables, through its influence on the distribution of currency across the economy. It will be seen that, following such a shock, the economy will converge over time to a steady state. However, a main result (Proposition 3) is that for certain parameter values, a monetary shock always reduces the economy's real GDP over the course of its transition to the steady state. In other words, the economy's GDP is at its global maximum when it is in its steady state. Although the proof of this assertion holds only for a narrow range of parameter values, evidence from numerous simulations, as well as the nature of the proof itself, strongly suggest that the result is true in general.

If the monetary shock is small, its impact can be examined by considering a linearization of the equilibrium in the neighbourhood of the steady state. This procedure indicates that small monetary shocks have no effect on aggregate output (Proposition 5). They do, however, alter the distribution of output. Since the distributional effects of a shock appear extremely complicated, it is useful to develop an approximation to the distribution that is somewhat more transparent. Thus Proposition 6 asserts that a linear combination of a reasonably small set of eigenvectors from the matrix $A$ closely approximates the distribution of production across the economy for all time periods beyond some $T>0$. An example shows that even for a very large economies, $T$ can be surprisingly small.

The paper also shows that the rate at which an economy returns to its steady state following a monetary shock depends importantly on its spatial structure. Specifically, different spatial structures can be associated with drastically different rates of convergence following a shock. This implies that an economy's spatial structure is part of the link that connects a change in monetary policy monetary to macroeconomic fluctuations. For instance, if the spatial structure is such that the economy returns rapidly to its steady state in the wake of a shock, then monetary factors are less likely to be an important source of business cycles. It appears clear that fluctuations in U.S. output have become less volatile since the early 1980's, a phenomenon which has come to be known as the "Great Moderation." "This paper suggests that changes in the spatial structure of the

\footnotetext{
${ }^{1}$ Blanchard and Simon, [8], compute standard deviations of quarterly output in a rolling window covering 20 quarters, over the period 1952-2000. They find that the standard deviation falls by a factor of three. McConnell and Perez-Quiros, [?], observe that the variance of output fluctuations during 1953:2-1983:4 exceeds the variance during 1984:1-1999:2 by a factor of four. Simon and Blume argue that output volatility has trended downwards since the early 1950's, with a significant deviation during
} 
economy - specifically, the density of inter-market linkages - could be a contributing factor to the reduction in output volatility.

The outline of the paper is as follows. Section 2 describes the model. Section 3 is concerned with the equilibria of the model. As might be expected for an overlapping generations model, there exists an uncountable infinity of equilibria. However, all but one of these is sensible as an economic equilibrium. This "fundamental equilibrium" is the focus of the remainder of the paper. Section 4 presents a combination of simulations and propositions that describe the effect of a monetary shock. Section 5 presents a range of examples that are intended to demonstrate the importance of the spatial structure in determining the rate at which an economy returns to a steady state following a monetary shock. Section 6 summarizes the basic mathematical machinery that facilitates the analysis of the model, and proofs are gathered in Section 7.

\subsection{Related Literature}

There are interesting similarities between the model introduced in this paper, and that of Grossman and Weiss, [18]. In that paper, each household makes cash withdrawals from a bank in alternating periods; the cash withdrawn in a particular period must satisfy the household's liquidity needs until it makes its next withdrawal, two periods hence. There are two cohorts of identical agents, one of which makes withdrawals in even periods, and one which makes withdrawals in odd periods. The cash received by a firm in exchange for consumption goods is presumed to flow directly into the bank, from where it is immediately withdrawn by the cohort whose turn it is to make a withdrawal. Thus all cash is always held by households. In a steady state money circulates between the two cohorts at rates that are determined by the parameters of the model. However, in the case where agents preferences are logarithmic, flows of cash between the two cohorts are described by the graphs in Figure BLANK, where each cohort is identified with one of the vertices, and where $0<\alpha<1$. In an odd period, members of one cohort spend a fraction $1-\alpha$ of their cash on consumption goods, and save $\alpha$ for consumption in the following period, while members of the other cohort spend all of their cash on consumption. The same sequence of transaction transpires in the subsequent period, but with the roles of the two cohorts reversed. Importantly, the value of $\alpha$, is invariant over time. If the economy is left undisturbed the distribution of cash across the two cohorts is eventually uniform. Grossman and Weiss assume that an expansionary monetary

the 1970's, while McConnell and Perez-Quiros find a structural break in the volatility of GDP growth in 1984:1. For further discussion of the phenomenon of reduced output volatility, as well as various candidate theories, see [5] and [27]. 
policy is executed by means of a lump sum cash transfer to the bank, which then flow immediately to the cohort whose withdrawal is scheduled for that period. Therefore, starting from a steady state, an expansionary monetary policy alters the distribution of cash across the two cohorts. But since the flow parameter $\alpha$ is fixed, prices must adjust so to maintain equilibrium in the goods market while accommodating the change cash flows. By assumption there is no production - one unit of output is supplied inelastically to the market in every period - therefore, a change in the distribution of cash across the two cohorts must induce a change in the distribution of consumption. Therefore, monetary policy is non-neutral, even though prices are perfectly flexible, and even though there are no stochastic elements.

The same basic phenomenon occurs in this paper. In a steady state, cash circulates through the economy at uniform rates. If a monetary shock alters the distribution of cash, then prices must adjust to accommodate the change in the volumes of cash flowing across the vertices. However, unlike the model in [18], production at each vertex is determined by relative prices in the relevant neighbourhood. Therefore, a monetary policy that alters the distribution of cash across the economy will, in general, induce a change in aggregate output through its effect on relative prices. Also, since this paper's focus is on the spatial structure of the economy, it allows for a far greater variety in underlying graph.

The non-neutrality of a monetary shock in this paper is due to two fundamental "frictions." The first is that the shock comes in the form of monetary transfers that are not uniform over agents. The second is that not all markets are directly linked. The first of these is also the basic friction in [18], and is discussed in the previous paragraph. The second of these frictions is primarily responsible for creating the liquidity effect manifested by a change in monetary policy in papers by Lucas, [23], and Fuerst, [16] (see also Christiano and Eichenbaum, [15]). For example, in the paper of Fuerst, there is an intra-period separation of the market for the consumption good from the market for loanable funds. Money is injected into the economy by means of transfers into the latter market. Due to the separation between markets, money that is newly injected into the market for loanable funds in any given period does not flow into the goods market until the following period. This fact is crucial in generating the non-neutrality of a change in monetary policy, for if money were injected in both markets simultaneously, households could act so as to undo the actions of the monetary authority.

A paper that explicitly incorporates spatial heterogeneity among agents is the wellknown "Turnpike model" of Townsend, [28]. In that paper two populations of agents travel in opposite directions along a linear turnpike. Each agent takes a discreet step in a fixed direction in each time period, such that each agent encounters one member of the opposing population in every period. Each of these encounter entails an exchange of an endowed good for money. Townsend is concerned with whether the presence of money 
enhances the welfare properties of the model's steady states.

It has been established that monetary policy can have real economic effects if agents are heterogeneous. Examples of this phenomenon are [6] and [?].

Finally, I like to think that the paper might be seen as formalizing the cash-in-advance constraint introduced in the seminal paper of Lucas, [22]. There Lucas motivates the cashin-advance constraint by supposing that each household produces a good of a particular colour, but prefers to consume goods of many different colours. Thus a household sells the good it produces in order to generate the cash required to purchase the range of goods that it consumes. However, in the actual model, the relative price between any two goods of different colours is one. In other words, the idea of differentiating among goods according to their colour is purely for motivational purposes. The framework proposed by Lucas is precisely the one that is adopted in this paper, although in an overlapping generations context: instead differentiating goods according to their colours, households differentiate goods according to their location.

\section{The Model}

The development of the model is divided into two subsections. The first describes the basic spatial structure, as well as the assumptions governing the individual agents. The second introduces more specialized assumptions about the spatial structure.

There is a finite set $G$ consisting of "locations" or "sites." As the elements of $G$ will be identified with the vertices of a graph, they will usually be referred to as such. A subset, $N(x)$, of $G$ is associated to each $x \in G$; this is the neighbourhood of $x$, and the agents inhabiting vertices in $N(x)$ are the neighbours of an agent at $x$. An agent at $x$ sells her output to old agents in $N(x)$ when she is young, and buys consumption goods from young agents in $N(x)$ when she is old (so clearly, if $y \in N(x)$, then $x \in N(y)$ ). Note that this eliminates the possibility of a more general structure whereby an agent at $x$ sells her output to one set of neighbours, and buys goods from another set of neighbours, where these two sets could be either overlapping or disjoint. However, such a structure would not alter the main results in a qualitatively meaningful way. Note that I am not claiming that this richer structure is completely benign; only that its effect is mostly on issues that are not the primary focus of the paper. An assumption that will be motivated in greater detail in the following section, is that the set $G$ is presumed to be a finite group. ${ }^{2}$ ) Thus a finite group $G$, together with a neighbourhood structure $\{N(x) \subset G \mid x \in G\}$,

\footnotetext{
${ }^{2}$ That is, $G$ is a non-empty set with a binary operation, usually written as multiplication, with the properties that $(a)(x y) z=x(y z),(b)$ there exists an element $e$, the identity, satisfying $x e=e x=x$ for all $x \in G$, and $(c)$ For each $x \in G$ there is a $x^{-1} \in G$ such that $x x^{-1}=x^{-1} x=e$.
} 
constitutes the economy's spatial structure. The spatial structure can be identified with an undirected graph, whose vertices are the elements of $G$, and whose edges are the unordered pairs $(x, y) \in G \times G$ satisfying $y \in N(x)$. Indeed, the graph itself will often be referred to as the economy's spatial structure. For example, the vertices of the graph in Figure 1 are labeled as $v_{0}, v_{1}, \ldots, v_{7}$, and $N\left(v_{0}\right)=\left\{v_{1}, v_{3}, v_{4}\right\}$. Some additional, more specialized assumptions will be imposed upon the structure of the graphs in Subsection 2.3 .

I now describe the agents in the model. Take as fixed a spatial structure, $G$ and $\{N(x) \subset G \mid x \in G\}$. Time is discrete and begins in period 1. Agents live for two periods; they are "young " in the first period of their lives, and "old" in the second period. A new generation of agents is born in each time period. Each new generation consists of $|G|$ agents, $^{3}$ with a young agent being born at each location in $G$. Therefore, at any moment in time, each site in $G$ is inhabited by two agents, one young and one old. (In period $t$ a site is the home of an old agent born in $t-1$, and a young agent born in $t .^{4}$ ) Agents produce when young and consume when old. However, agents do not consume the goods that they produce themselves; instead each agent sells her output in exchange for cash, which she then uses to purchase consumption goods in the following period. To be precise, the agent born at vertex $x$ in period $t$ produces output in her first period of life, and sells it to the old agents located at the vertices in $N(x)$ in exchange for cash. This cash is then carried forward into period $t+1$, where the agent uses it to purchase goods produced by the young agents located in $N(x)$. All transactions are mediated by money which is presumed to be both inconvertible and intrinsically useless. All goods that are not consumed are assumed to perish immediately following the period during which they are produced. Also, we make the usual overlapping generations assumption that there is a generation of old agents, one at each vertex, already living when time begins in period 1 .

The preferences of the agent born in period $t$ at vertex $x$ are described by the utility function

$$
|N(x)|\left(\prod_{y \in N(x)} c_{t+1}(x, y)\right)^{\frac{1}{|N(x)|}}-L_{t}(x)^{\alpha}
$$

where $L_{t}(x)$ is the quantity of labour she supplies in period $t$ (when she is young), and $c_{t+1}(x, y)$ is the quantity of the consumption good she purchases from the young agent

\footnotetext{
${ }^{3}$ If $X$ is a set, then $|X|$ denotes the number of elements in $X$.

${ }^{4}$ Certainly other demographic arrangements are possible: For instance, it might seem more natural for each vertex to be inhabited by a single agent. However, the framework adopted here has the virtue of being "demographically homogeneous," in that every vertex is occupied by a young and an old agent in all periods. This is a convenient simplification that does not seem to have significant costs.
} 
at $y$ in period $t+1$ (when she is old). Also, $\alpha$ is a constant satisfying $\alpha>1$. Two slightly anomalous aspects of this specification are 1). the utility of consumption is not discounted relative to the disutility of labour in the previous period, and 2). the utility of consumption is multiplied by the constant $|N(x)|$. The only effect of these facts is to reduce the number of inert constants carried from one formula to the next.

This utility function is central to the paper; it has certain basic implications from which many, if not all, of the basic results flow. Its first fundamental implication is that money flows through the economy according to a reversible Markov process. This both simplifies and clarifies the analysis at every point in the paper. The second fundamental implication (which, in many respects, is a consequence of the first) is that for large classes of graphs, the economy's equilibrium can be expressed as closed-form functions of the exogenous data. This means, among other things, that intelligible statements can be made about the effects of a monetary shock. For instance, both rigorous proofs and precise calculations are obtainable. The third implication is that the flow of money through the economy will be naturally compatible with the group operation associated with $G$. This renders the economy's dynamics amenable to being analyzed by powerful methods from Fourier analysis.

The young agent located at vertex $x$ possesses the constant returns to scale production function

$$
q_{t}(x)=L_{t}(x)
$$

where $q_{t}(x)$ is the quantity of the good produced by the young agent at $x$ in period $t$. This production function is shared by all agents both across the economy and over time.

The symbol $m_{t}(x)$ represents the number of units of currency held by the old agent at vertex $x$ at the outset of period $t$, and $m_{t} \equiv\left\{m_{t}(x) \mid x \in G\right\} \in \mathbb{R}^{|G|}$ is the distribution of money in period $t$. Monetary policy is executed by means of transfers to agents at various locations across the economy. It will be assumed that unanticipated transfers are made to old agents at the outset of period 1 only. Thereafter the monetary authority sits idle for all time. In other words, the sole act of the Central Bank is to choose the initial distribution of money, $m_{1}$, subject to the single requirement that $m_{1}>0$. The effects of this "shock" on the economy is the entire focus of the paper.

In all periods except the first, the cash balances held by the old agent at $x \in G$ in any period are obtained as the proceeds from the sale of her output in the previous period. Therefore,

$$
m_{t+1}(x)=p_{t}(x) q_{t}(x), \quad t=2,3, \ldots
$$

where $p_{t}(x)$ is the price of the good produced at vertex $x$ in period $t$. (As mentioned, $m_{1}$ is determined exogenously by the Central Bank.) The budget constraint faced by the 
old agent at $x$ in period $t+1$ is

$$
\sum_{y \in N(x)} p_{t+1}(y) c_{t+1}(x, y)=m_{t+1}(x) .
$$

All agents are price takers, and are endowed with perfect foresight, so their decisions are made with knowledge of all relevant prices.

So altogether, in period $t$ the agent inhabiting vertex $x$ chooses $L_{t}(x), q_{t}(x)$, and $c_{t+1}(x, y), y \in N(x)$, so as to maximize (1), subject to the constraints (2), (3), and (4). The solution is

$$
\begin{aligned}
q_{t}(x) & =\gamma\left(\frac{p_{t}(x)}{\prod_{y \in N(x)} p_{t+1}(y)^{\frac{1}{|N(x)|}}}\right)^{\frac{1}{\alpha-1}} \\
L_{t}(x) & =q_{t}(x) \\
c_{t+1}(x, y) & =\frac{m_{t+1}(x)}{|N(x)| \cdot p_{t+1}(y)}, \quad \text { for all } y \in N(x) .
\end{aligned}
$$

where $\gamma$ is written in place of the constant $\left(\frac{1}{\alpha}\right)^{\frac{1}{\alpha-1}}$. Cobb-Douglas preferences imply that each agent spends $\frac{1}{|N(x)|}$ of her income on each of the $|\Omega(x)|$ goods that she consumes. The quantity of output she produces depends (positively) on the price of her own good relative to the geometric average of the prices of the goods she buys.

Finally, the economy's nominal GDP in period $t$ is $\sum_{x \in G} p_{t}(x) q_{t}(x)$. However, in equilibrium, total output is exchanged for the total money stock in each period (that is, the velocity of money is always one), so nominal GDP in period $t$ is also equal to $\sum_{x \in G} m_{t}(x)$.

\subsection{Monetary Policy}

The paper is concerned with a basic experiment whereby the Central Bank executes unanticipated lump sum transfers to old agents at the outset of period 1. The paper then investigates the effect of this "shock" as it diffuses through the economy. The Central Bank takes no other action in any other time period. In other words, the monetary authority's sole action is to choose the distribution of money $m_{1}$ that prevails at the outset of period 1. Any distribution is admissible provided only that $m_{1}(x)>0$ for all $x \in G$. Let $M$ denote the total stock of cash in the economy $M=\sum_{x \in G} m_{1}(x)$. This stock of currency remains forever in circulation (following period 1), being neither added to nor subtracted from. 


\subsection{Graphs and the Spatial Structure}

This subsection gives a more detailed description of the graphs that comprise the model's spatial structure. Let $G$ be a finite group with identity $e$, and let $\Omega=\left\{\omega_{1}, \ldots, \omega_{k}\right\}$ be a fixed subset of $G$. For reasons that will become clear, $\Omega$ is referred to as a generating set. The structure of the graphs in the paper are implied by the following assumptions:

Assumption G0: For each $x \in G$, the neighbourhood of $x$ is defined according to

$$
N(x)=\{y \in G \mid x \omega=y \text { for some } \omega \in \Omega\}
$$

Assumption G1: e $\notin \Omega$.

Assumption G2: $\Omega$ is symmetric. That is, $\omega \in \Omega$ if and only if $\omega^{-1} \in \Omega$.

Assumption G3: Every $x \in G$ can be expressed as a product of members of $\Omega$. That is, for each $x \in G$, there are $\omega_{i_{1}}, \ldots, \omega_{i_{n}} \in \Omega$, such that $x=\omega_{i_{1}} \omega_{i_{2}} \cdot \ldots \cdot \omega_{i_{n}}$

Assumption $G_{4}$ : $\Omega$ is the union of conjugacy classes of $G$.

Assumption $G 0$ describes the neighbourhood structure of the graph. It states that, for any $x \in G$, the neighbourhood $N(x)$ is obtained by subjecting $x$ to right multiplication by the elements of $\Omega$. It is both very strong and very important, for it implies that all neighbourhoods have an identical structure. That is, from the perspective of an occupant of any vertex, each neighbourhood is indistinguishable from every other neighbourhood. Assumption $G 1$ states that no $x$ is a neighbour of itself. Assumption $G 2$ guarantees that $y \in N(x)$ if and only if $x \in N(y)$. Technically, it implies that the associated graph is undirected. Assumption $G 3$ ensures that the graph is connected, meaning that for any two vertices $x, y \in G$ there is a path (a sequence of edges) running from $x$ to $y$. Without it, the economy could be composed of a collection of disjoint components, each constituting an isolated economy.

Unlike Assumptions $G 0-G 3$, the implications of $G 4$ for the structure of the graph are not at all obvious. In fact, I am unaware of any interpretation, either economic, or graph-theoretic. The assumption has been added to the list of requirements, even though it is not used to generate any of the formal results of Sections 3-5. Instead its role is to facilitate the computations in many of the examples. If the underlying group is abelian, then Fourier methods are ideal for extracting eigenvalues and eigenvectors of the graph.

However, the same methods are, in general, not available if the underlying group is not abelian. However, if the set $\Omega$ is the union of conjugacy classes, then some of the very desirable properties of Fourier analysis on abelian groups to be exported intact 
to the setting of a non-abelian group. Consequently, a complete eigenvalue/eigenvector decomposition of $\delta_{\Omega}$ (defined below) can be readily obtained, even in the case of some very large economies. This fact is described formally in a theorem stated in Section 6 . Examples BLANK, Tables BLANK, and Figures BLANK are all based on eigenvalues and eigenvectors that have been obtained through the method indicated by the theorem. The content of the theorem is well-known to mathematicians who work with the theory of representation of groups. Its powerful implications were, as far as I know, first recognized by Persi Diaconnis, who exploits it to generate surprisingly precise estimates of the rate at which certain card-shuffling algorithms generate a random deck. ${ }^{5}$ A final point here is that $G 4$ is potentially quite strong in that for many groups, the smallest generating set that satisfies both $G 2$ and $G 4$ may be large relative to the group. For example, the smallest such generating set for the dihedral groups contain more than half the group's members.

Thus the spatial structure of the model is identified with a graph built from a finite group $G$, together with a generating set $\Omega$ satisfying assumptions $G 0-G 3$. The vertices are the elements of $G$, and the edges are the pairs $(x, y) \in G \times G$, where $y=x \omega$ for some $\omega \in \Omega$. Such graphs are widely known as Cayley graphs. The symbol $\Gamma(G, \Omega)$ will denote one such. The following examples will further illustrate the concept of a Cayley. The first three recur throughout the paper, and the fourth is interesting for its own sake.

Example 2.1: The cycle graph on $n$ vertices (denoted $C_{n}$ ): This is the Cayley graph whose underlying group is the cyclic group of order $n$. This group is identified with the set of integers $\bmod n$, denoted by $\mathbb{Z}_{n}$, under the group operation of addition $(\bmod n)$. The generating set is $\Omega=\{-1,1\}$ (once again, $\bmod n$ ). Figure 2.a shows the cycle graphs on 8 vertices.

Example 2.2: The $n$-dimensional hypercube, (denoted $H_{n}$ ): Let $\mathbb{Z}_{2}$ denote the two-element abelian group whose elements are $\{0,1\}$, under the group operation of addition mod 2 , and let $\mathbb{Z}_{2}^{n}$ be the $n$-fold direct product (that is, $\mathbb{Z}_{2} \times \mathbb{Z}_{2} \times \ldots \times \mathbb{Z}_{2}$ with $n$ factors). ${ }^{6}$ Also, let $e_{i} \in \mathbb{Z}_{2}^{n}$ be the $n$-tuple with a 1 in the $i^{\text {th }}$ position, and 0's elsewhere. Then the hypercube is the Cayley graph whose underlying group is $\mathbb{Z}_{2}^{n}$, and whose generating set is $\Omega=\left\{e_{1}, \ldots, e_{n}\right\}$. In words, each vertex is identified with an $n$-vector of 0 's and 1's,

\footnotetext{
${ }^{5} \mathrm{~A}$ summary of the remarkable properties of particular shuffling algorithms (with particular emphasis on the "cut-off phenomenon") are described in [14]. Further mathematical detail is provided in [13] and [24].)

${ }^{6}$ Thus $\mathbb{Z}_{2}^{n}$ consists of all ordered $n$-tuples, $\left(x_{1}, \ldots, x_{n}\right)$, where $x_{i} \in\{0,1\}$ for $i=1, \ldots, n$, and where the group operation is component-wise addition mod 2. That is, $\left(x_{1}, \ldots, x_{n}\right)+\left(y_{1} \ldots, y_{n}\right)=$ $\left(x_{1}+y_{1}, \ldots, x_{n}+y_{n}\right)$, where all sums are mod 2 .
} 
and the neighbours of any vertex are those vectors which differ from the given vertex in one digit only. (So for instance, in $H_{4}$, the vertex $(0,1,0,0)$ is adjacent to the vertices $(1,1,0,0),(0,0,0,0),(0,1,1,0)$, and $(0,1,0,1)$. The graph $H_{n}$ has $2^{n}$ vertices, $n 2^{n-1}$ edges, and each vertex has $n$ neighbours. The graph $H_{3}$ is shown in Figure 1.

Example 2.3: The transposition graph, ${ }^{7}$ (denoted $\Gamma\left(S_{n}, T_{n}\right)$ ): The symmetric group $S_{n}$ is the group whose elements are the bijections from the set $\{1,2, \ldots, n\}$ into itself, and where the group operation is the ordinary composition of functions. Let $T_{n}$ be the set of transpositions in $S_{n}$, where an element $\tau \in S_{n}$ is a transposition if it interchanges two elements of $\{1, \ldots, n\}$, while leaving all other elements fixed. ${ }^{8}$ Then the transposition graph is the Cayley graph with group $S_{n}$, and generating set $T_{n}$. Thus each vertex is a permutation of the integers $\{1, \ldots, n\}$, and the elements adjacent to a permutation $\left(i_{1}, i_{2}, \ldots, i_{n}\right)$ are those permutations that are obtained from $\left(i_{1}, \ldots, i_{n}\right)$ by interchanging (transposing) any two integers. The graph has $n$ ! vertices, and each vertex has $\left|T_{n}\right|=\left(\begin{array}{l}n \\ 2\end{array}\right)$ neighbours, and there are a total of $\frac{n !}{2}\left(\begin{array}{l}n \\ 2\end{array}\right)$ edges.

Example 2.4: The Buckeyball: Figure 2.b shows the Buckyball, which is obtained as a Cayley graph built on the alternating group $A_{5},{ }^{9}$ with the generating set $\Omega=\{(12345),(54321),(12)(34)\}$.

The final task of the subsection is to introduce the concept of an adjacency matrix, which will play a prominent role throughout the paper. Let $\Gamma(V, E)$ be an arbitrary undirected graph, with vertex set $V$ and edge set $E$. The adjacency matrix corresponding to the graph $\Gamma(V, E)$, is a $|V| \times|V|$ matrix $A_{\Gamma}$ whose rows and columns are both indexed by the vertices of the graph, and where $A_{\Gamma}(i, j)=1$ if $v_{i}$ and $v_{j}$ are adjacent, and $A_{\Gamma}(i, j)=0$ otherwise. (Here $A_{\Gamma}(i, j)$ is element in row $i$ and column $j$ of the matrix $A_{\Gamma}$.) The matrices $A_{\Gamma}$ for the graphs in Figures 1 and 2.a are

\footnotetext{
${ }^{7}$ This terminology is not standard.

${ }^{8}$ Formally, $\tau \in S_{n}$ is a transposition if $\tau(i)=j$ and $\tau(j)=i$ for some $i, j \in\{1, \ldots, n\}$ where $i \neq j$, and $\tau(k)=k$ for all $k \neq i$ and $k \neq j$.

${ }^{9}$ If $S_{n}$ is the group of all permutations of $n$ objects, then $A_{n}$ is the subset of $S_{n}$ consisting of even permutations. The group $A_{5}$ can also be realized as the group of rotational symmetries of a dodecahedron (see Section 6.2 of [2]) Also, the elements of the generating set are described in "cycle notation," a description of which will be contained in any algebra text. (See, for instance, [2], page 213, or the section "Permutation Groups," of [19].)
} 


\begin{tabular}{|c|c|c|c|c|c|c|c|c|}
\hline & $v_{0}$ & $v_{1}$ & $v_{2}$ & $v_{3}$ & $v_{4}$ & $v_{5}$ & $v_{6}$ & $v_{7}$ \\
\hline ר & 0 & 1 & 0 & 1 & 1 & 0 & 0 & 0 \\
\hline$v$ & 1 & 0 & 1 & 0 & 0 & 1 & 0 & 0 \\
\hline$v_{2}$ & 0 & 1 & 0 & 1 & 0 & 0 & 1 & 0 \\
\hline & 1 & 0 & 1 & 0 & 0 & 0 & 0 & 1 \\
\hline & 1 & 0 & 0 & 0 & 0 & 1 & 0 & 1 \\
\hline$v$ & 0 & 1 & 0 & 0 & 1 & 0 & 1 & 0 \\
\hline & 0 & 0 & 1 & 0 & 0 & 1 & 0 & 1 \\
\hline & 0 & 0 & 0 & 1 & 1 & 0 & 1 & 0 \\
\hline
\end{tabular}

\begin{tabular}{ll}
\multirow{4}{*}{ and } & $v_{0}$ \\
& $v_{1}$ \\
& $v_{2}$ \\
& $v_{3}$ \\
& $v_{4}$ \\
& $v_{5}$ \\
& $v_{6}$ \\
& $v_{7}$
\end{tabular}$\left(\begin{array}{cccccccc}v_{0} & v_{1} & v_{2} & v_{3} & v_{4} & v_{5} & v_{6} & v_{7} \\
0 & 1 & 0 & 0 & 0 & 0 & 1 & 0 \\
0 & 0 & 1 & 0 & 0 & 0 & 0 & 1 \\
1 & 0 & 0 & 1 & 0 & 0 & 0 & 0 \\
0 & 1 & 0 & 0 & 1 & 0 & 0 & 0 \\
0 & 0 & 1 & 0 & 0 & 1 & 0 & 0 \\
0 & 0 & 0 & 0 & 1 & 0 & 0 & 1 \\
1 & 0 & 0 & 0 & 0 & 1 & 0 & 0\end{array}\right)$

A graph can be constructed in its entirety from knowledge of its adjacency matrix. This implies that the adjacency matrix contains all of the information embedded in the associated graph. This fact is extremely useful, for it enables all of the properties of a graph to be expressed as an algebraic object.

\subsection{Motivation for Cayley Graphs}

I now motivate the use of a group as the basic ingredient in the model's spatial structure. There are three reasons for assuming that the underlying graph is of the Cayley variety.

The first reason is that Cayley graphs have a high degree of symmetry, which simplifies the analysis tremendously. This fact can be described in more formally through the concept of a graph automorphism. If $\Gamma(V, E)$ is an undirected graph with vertex set $V$ and edge set $E$, then an automorphism of $\Gamma(V, E)$ is a permutation $\varphi$ of the vertices, $\varphi: V \rightarrow V$ such that $(\varphi(x), \varphi(y))$ is an edge of $\Gamma(V, E)$ if and only if $(x, y)$ is also an edge of $\Gamma(V, E)$. Hence a graph automorphism reflects a symmetry in the structure of the graph. The set of automorphisms of a graph is a group under the operation of composition. Therefore, graphs with large automorphism groups are highly symmetric. There is a loose hierarchy of symmetry categories, with vertex transitive graphs being the largest category. A graph $\Gamma(V, E)$ is vertex transitive if for any two vertices $x, y \in$ $V$, there is an automorphism $\varphi$ such that $\varphi(x)=y$. Also, such graphs automatically have automorphism groups with at least as many elements as there are vertices in the graph. A main point here is that Cayley graphs comprise the largest category of vertex transitive graphs. They are also the most conspicuous, as they are easily constructed. Although there exist vertex transitive graphs that are not are Cayley graphs, they are neither common, nor easily identified. In other words, if one were to simply impose the symmetry condition that all neighbourhoods possess the same local structure, then one 
would simultaneously be restricting the graphs to a class (those that are vertex transitive) of which Cayley graphs are the most prominent members.

The second reason is that the diffusion of money through the economy is naturally compatible with the group operation that determines the neighbourhood structure of the graph. To see this fix a Cayley graph $\Gamma(G, \Omega)$, and let $m_{t}=\left\{m_{t}(x) \mid x \in G\right\} \in \mathbb{R}^{|G|}$ denote the distribution of money in period $t$. Note that $|N(x)|=|\Omega|$ for all $x \in G$. The utility function (1) implies that in every period the old agent at any vertex $y$ divides her cash holdings into $|N(y)|$ equal portions, and then "sends" each of these portions to a neighbour in $N(y)$ in exchange for consumption goods. Therefore, in period $t$, the young agent at any vertex $x$ receives the fraction $\frac{1}{|N(y)|}$ of the cash balances held by each old agent $y \in N(x)$; and since these are the cash balances that she brings forward into period $t+1$, it follows that $m_{t+1}(x)=\frac{1}{|N(y)|} \sum_{y \in N(x)} m_{t}(y)$; then, in terms of the notation introduced above,

$$
m_{t+1}(x)=\frac{1}{|\Omega|} \sum_{\omega \in \Omega} m_{t}\left(x \omega^{-1}\right) .
$$

Next let $G$ be a finite group, and note that $\mathbb{C}^{|G|}$ corresponds to the set of complexvalued functions on $G$. The group operation on $G$ induces a binary operation $*$ on $\mathbb{C}^{|G|}$, known as the convolution product. Specifically, for any $f, g \in \mathbb{C}^{|G|}$ the convolution of $f$ and $h$ is defined according to

$$
f * h(x)=\sum_{y \in G} f\left(x y^{-1}\right) h(y)
$$

This concept has a rich heritage in mathematics, for it plays a central role in Fourier analysis (See $[21]$ for instance.) Now define the function $\delta_{\Omega}: G \rightarrow \mathbb{R}$ by $^{10}$

$$
\delta_{\Omega}(x)= \begin{cases}\frac{1}{|\Omega|} & \text { if } x \in \Omega \\ 0 & \text { if } x \notin \Omega\end{cases}
$$

\footnotetext{
${ }^{10}$ This is an abuse of a standard notation according to which the indicator function $\delta_{A}$ takes the value 1 on the set $A$ and 0 on its compliment. Here $\delta_{\Omega}$ takes the value $\frac{1}{|\Omega|}$ on $\Omega$ rather than 1 .
} 
Then

$$
\begin{aligned}
m_{t} * \delta_{\Omega}(x) & =\sum_{y \in G} m_{t}\left(x y^{-1}\right) \delta_{\Omega}(y) \\
& =\sum_{\omega \in \Omega} m_{t}\left(x \omega^{-1}\right) \delta_{\Omega}(\omega) \\
& =\frac{1}{|\Omega|} \sum_{\omega \in \Omega} m_{t}\left(x \omega^{-1}\right) \\
& =m_{t+1}(x) .
\end{aligned}
$$

Thus the evolution of the distribution of money from one period to the next is governed by convolution with the fixed function $\delta_{\Omega}$. In words, given a function $f \in \mathbb{R}^{|G|}$, the convolution operator produces a new function whose value at $x$ is the average of the values taken by $f$ in the neighbourhood of $x$. But Cobb-Douglas preferences imply that this is exactly the process according to which the distribution of money evolves from one period to the next. This is the second reason for using a Cayley graph: The flow of money through the economy is described by the convolution product, which is a binary operation on $\mathbb{C}^{|G|}$ which, in turn, is naturally induced by the operation on the underlying group $G$.

The third reason is that some very demanding computations are greatly simplified by the linear structure that can be built from the group. If the underlying group is abelian, then the eigenvalues and eigenvectors for the operator $\delta_{\Omega}$ can be obtained by elementary methods of Fourier analysis. And if the group is not abelian, then the same methods can be successfully generalized in order to deliver the eigenvalue/eigenvector decomposition of $\delta_{\Omega}$, provided that assumption $G 4$ holds. As mentioned above, these methods are exploited in most of the examples in the paper.

Remark 1: It should be pointed out that the flow of money through the economy can also be viewed as a reversible Markov process. In any time period $t$, think of a unit of currency at a vertex $x$ as moving to any one of the vertices in $N(x)$ with probability $\frac{1}{|\Omega|}$. For an economy whose spatial structure is furnished by a graph $\Gamma(G, \Omega)$, with adjacency matrix $A_{\Gamma}$, the corresponding Markov matrix is $\frac{1}{|\Omega|} A_{\Gamma}$. Since this matrix will be referred to frequently in the sequel, it will be designated as $A$, and referred to colorfully as the diffusion matrix. Thus, $m_{t+1}=\frac{1}{|\Omega|} A_{\Gamma} m_{t} \equiv A m_{t}$ for all $t$.

Remark 2: I have now introduced two equivalent notations to describe the same phenomenon, namely $m_{t+1}=\delta_{\Omega} * m_{t}$ and $m_{t+1}=A m_{t}$. These simply reflect a single set of 
objects as seen from two different perspectives. In the former notation, $\delta_{\Omega}$ is an operator on $\mathbb{R}^{|G|}$, where $\mathbb{R}^{|G|}$ is regarded as the set of complex-valued functions on $G$, while, in the latter notation, $A$ is a function on the same set $\mathbb{R}^{|G|}$, but where $\mathbb{R}^{|G|}$ is regarded as a vector space over $\mathbb{R}$. Both notations will occur later in the paper.

\section{Monetary Shocks 1: Competitive Equilibrium}

The economy described in the previous section has a unique "sensible" equilibrium. This section provides a formal statement of this fact, and then characterizes this equilibrium. Its properties are investigated in the next section.

Given a Cayley graph $\Gamma(G, \Omega)$, and an initial distribution of cash balances $m_{1}>0$, a competitive equilibrium is a set of sequences of positive functions

$$
p_{t}: G \rightarrow \mathbb{R}, \quad L_{t}: G \rightarrow \mathbb{R}, \quad q_{t}: G \rightarrow \mathbb{R}, \quad c_{t}: G \times G \rightarrow \mathbb{R}, \quad m_{t}: G \rightarrow \mathbb{R}
$$

$t=1,2, \ldots$, that satisfy both the individual optimality conditions (5) - (7), and the equilibrium condition

$$
q_{t}(x)=\frac{1}{|\Omega|} \sum_{y \in N(x)} \frac{m_{t}(y)}{p_{t}(x)}
$$

for all $t=1,2, \ldots$ and all $x, y \in G$. The equality (9) is simply the market-clearing condition for the good produced at vertex $x$ in period $t .^{11}$

The following proposition makes the point that although there are uncountably many equilibria, there is only one for which prices and quantities remain bounded. Moreover, in any equilibrium where prices and quantities are not bounded, there must be a subsequence of time periods along which either prices or quantities are, on average, exploding at a doubly exponential rate. Also, if prices are growing at a doubly exponential rate, then quantities must be shrinking at a doubly exponential rate, and vice versa. A double exponential growth rate in prices corresponds to an exponential growth rate in inflation, which, in turn, constitutes a hyperinflation. Since no economy with a constant money stock has ever produced a time series of this nature, (for growth or shrinkage at a double exponential rate is truly staggering), it does not seem unduly restrictive to rule out

\footnotetext{
${ }^{11}$ Equilibrium in the money market can be described as $\sum_{x \in G} \sum_{y \in N(x)} p_{t}(y) c_{t}(x, y)=M$, where the double sum on the lefthand side is the total cash required to carry out consumption goods across the economy in period $t$. This equality is not listed among the formal requirements for an equilibrium as it is implied by the other conditions.
} 
such equilibria. As a consequence, the paper focuses exclusively on the unique bounded equilibrium.

Proposition 1: Let $\Gamma(G, \Omega)$ be any Cayley graph, and let $m_{1}>0$ be any initial distribution of money. Then

1. There exists an uncountable infinity of equilibria.

2. There is a unique equilibrium for which prices, $\left\{p_{t} \in \mathbb{R}^{|G|} \mid t=1,2 \ldots\right\}$, and quantities, $\left\{q_{t} \in \mathbb{R}^{|G|} \mid t=1,2, \ldots\right\}$, are bounded. This is referred to as the fundamental equilibrium.

3. Let $\left\{p_{t} \in \mathbb{R}^{|G|} \mid t=1,2 \ldots\right\}$ and $\left\{q_{t} \in \mathbb{R}^{|G|} \mid t=1,2, \ldots\right\}$ be the prices and quantities in any equilibrium other than the fundamental equilibrium. There exists a subsequence of integers $\left\{n_{k}\right\}_{k=1}^{\infty}$ and an integer $K$ such that once $k>K$, at least one of (a) or (b) is true (Recall that $\alpha>1$.):

(a) There exist positive constants $c_{1}, \ldots, c_{4}$ such that

$$
c_{1} e^{c_{2} \alpha^{n_{k}}}<\frac{1}{|G|} \sum_{x \in G} p_{n_{k}}(x) \quad \text { and } \quad c_{3} e^{c_{4} \alpha^{n_{k}}}<\sum_{x \in G} \frac{1}{q_{n_{k}}(x)}
$$

(b) There exist positive constants $c_{5}, \ldots, c_{6}$, such that

$$
c_{5} e^{c_{6} \alpha^{n_{k}}}<\sum_{x \in G} \frac{1}{p_{n_{k}}(x)} \quad \text { and } \quad c_{7} e^{c_{8} \alpha^{n_{k}}}<\frac{1}{|G|} \sum_{x \in G} q_{n_{k}}(x)
$$

It has been emphasized that the model admits closed-form solutions for the prices and quantities in the fundamental equilibrium. These are stated in the following proposition. First, some notation: if $f \in \mathbb{R}^{|G|}$, with $f>0$, then $\log f$ is the real-valued function that takes the value $\log f(x)$ at $x \in G$. Also, let $A_{\Gamma}^{r}(i, j)$ denote the element of the matrix $A_{\Gamma}^{r}$ whose row index is $x_{i} \in G$, and whose column index is $x_{j} \in G$.

Proposition 2: Let $\left\{p_{t} \in \mathbb{R}^{|G|} \mid t=1,2 \ldots\right\}$ and $\left\{q_{t} \in \mathbb{R}^{|G|} \mid t=1,2, \ldots\right\}$ denote the 
prices and quantities in the fundamental equilibrium. Then

$$
\begin{gathered}
p_{t}\left(x_{i}\right)=\frac{1}{\gamma}\left(m_{t+1}\left(x_{i}\right) \prod_{j=1}^{\infty}\left(m_{t+j+1}\left(x_{1}\right)^{A^{j}(i, 1)} m_{t+j+1}\left(x_{2}\right)^{A^{j}(i, 2)} \cdot \ldots \cdot m_{t+j+1}\left(x_{|G|}\right)^{A^{j}(i,|G|)}\right)^{\frac{1}{\alpha^{j}}}\right)^{1-\frac{1}{\alpha}} \\
q_{t}\left(x_{i}\right)=\gamma\left(\prod_{j=1}^{\infty}\left(\frac{m_{t+1}\left(x_{i}\right)}{m_{t+j+1}\left(x_{1}\right)^{A^{j}(i, 1)} m_{t+j+1}\left(x_{2}\right)^{A^{j}(i, 2)} \cdot \ldots \cdot m_{t+j+1}\left(x_{|G|}\right)^{A^{j}(i,|G|)}}\right)^{\frac{1}{\alpha^{j}}}\right)^{1-\frac{1}{\alpha}}
\end{gathered}
$$

Before examining these expressions, it will be helpful to interpret the entries in the matrix $A_{\Gamma}^{r}$. (Recall that $A_{\Gamma}$ is the adjacency matrix, and that $A$ and $A_{\Gamma}$ are related according to $A=\frac{1}{|G|} A_{\Gamma}$.) For a graph $\Gamma(G, \Omega)$, define a walk of length $r$ from $x_{i} \in G$ to $x_{j} \in G$ to be a sequence of vertices $x_{i}=v_{h_{1}}, v_{h_{2}}, \ldots, v_{h_{r}}=x_{j}$, where $v_{h_{k}}$ and $v_{h_{k+1}}$ are adjacent. A basic fact is that $A_{\Gamma}^{r}(i, j)$ equals the number of walks of length $r$ from vertex $x_{i}$ to vertex $x_{j}$. (see Lemma 2.5, page 9 of [7]).

From BLANK it can be seen that the price at $x$ in period $t$ is higher if 1 ). cash holdings of the neighbours of $x$ in period $t+1$ are higher, and 2). the prices of the neighbours of $x$ are higher in $t+1$. Let $y_{1}, \ldots, y_{k}$ be the vertices adjacent to $x$. Higher prices at $y_{1}, \ldots, y_{k}$ in period $t+1$ induce the agent at $x$ to produce a smaller quantity of output in period $t$ (this can be seen from the expression BLANK); this reduced supply, in turn, drives $p_{t}(x)$ up. Also, this dependence of current prices on future prices iterates indefinitely into the future. This explains the terms $m_{t+j+1}\left(x_{k}\right)^{A^{j}(i, k)}$ in BLANK: The influence of cash held by the neighbours of a vertex $x_{j}$ in period $t+r+1$ on the price at $x_{i}$ in period $t$ is reflected in the weight $A^{r}(i, j)$, which is the number of ways in which $x_{j}$ is a neighbour of a neighbour of ... a neighbour of $x_{i}$, (where the dots here are intended to indicate $r$ repetitions of the phrase "a neighbour of").

Equation BLANK it can be seen that an agent's supply of output increases as the price of her output increases relative to the prices of the goods she consumes. Accordingly, $q_{t}(x)$ depends positively on the cash holdings of the neighbours of $x$ in $t$ relative to the cash holdings of agents who are neighbours of the neighbours of $x$ in $t+1$. However, like $p_{t}(x)$, the fact that $q_{t}(x)$ depends on prices at neighbouring vertices in $t+1$, implies that it also depends on future cash holdings at all vertices, to a degree that is reflected in the combinatorial structure of the graph.

The expressions (10) and (11) show that the equilibrium is "forward looking," in that, in any given period, the prices and production at all locations in the economy depend only on the distribution of money over all future periods. One implication is that, in 
general, prices and quantities in all locations respond immediately to a monetary shock. Another implication is that "history does not matter;" in particular, in period $t \geq 1$, it is irrelevant whether the initial monetary shock was a large expansionary policy or, at the opposite extreme, a large contractionary policy.

Although it may not be immediately evident, the equilibrium manifests the usual dichotomy between real and nominal variables, insofar as prices and quantities in any period are homogeneous of degree one and zero, respectively, in the initial distribution, $m_{1}$.

Another point regarding the fundamental equilibrium concerns the asymptotic behavior of prices and quantities. As $t$ goes to infinity, prices and quantities converge to one of two possible steady states, one where prices are uniform across the economy and stationary over time, and the other where all prices oscillate between two values form one period to the next. Accordingly, I will refer to the former as a stationary steady state, and to the latter as a cyclical steady state. Cyclical steady states occur only if the matrix $A$ has an eigenvalue equal to -1 , and this, in turn, occurs if and only if the corresponding graph is bipartite. A fact which is perhaps surprising is that even though prices oscillate in a cyclical steady state, quantities do not. The following corollary embodies these points. Its formal statement requires a modest build-up of notation.

If $\Gamma(G, \Omega)$ is bipartite then $G$ can be partitioned into two (disjoint) subsets, $G_{1}$ and $G_{2}$, such that if $x$ is any vertex in $G_{i}$, then every neighbour of $x$ is in $G_{j}, i, j \in\{1,2\}$, $i \neq j{ }^{12}$ Let the labels on $G_{1}$ and $G_{2}$ be chosen such that the identity, $e$, belongs to $G_{1}$. Also, in the event that $\Gamma(G, \Omega)$ is bipartite, let $M_{i}, i=1,2$, denote the total cash balances held by old agents in $G_{i}$ in period $1, i=1,2$. That is $M_{i}=\sum_{x \in G_{i}} m_{1}(x)$, $i=1,2$. (Recall that $M$ is the total cash in the economy.)

Corollary: Let $\left\{p_{t} \in \mathbb{R}^{|G|} \mid t=1,2 \ldots\right\}$ and $\left\{q_{t} \in \mathbb{R}^{|G|} \mid t=1,2, \ldots\right\}$ denote the prices and quantities in the fundamental equilibrium. If $\Gamma(G, \Omega)$ is not bipartite, then

$$
\lim _{t \rightarrow \infty} p_{t}(x)=\frac{M}{|G| \gamma} \quad \text { and } \quad \lim _{t \rightarrow \infty} q_{t}(x)=\gamma
$$

for all $x \in G$. If $\Gamma(G, \Omega)$ is bipartite, then $\lim _{t \rightarrow \infty} q_{t}(x)=\gamma$ for all $x \in G$, and for any $x \in G_{1}$ and any $y \in G_{2}$

$$
\begin{aligned}
& \lim _{t \rightarrow \infty, t \text { even }} p_{t}(x)=\lim _{t \rightarrow \infty, t \text { odd }} p_{t}(y)=\frac{1}{\gamma|G|}\left(\left(M+M_{1}-M_{2}\right)^{\alpha}\left(M+M_{2}-M_{1}\right)\right)^{\frac{1}{1+\alpha}} \\
& \lim _{t \rightarrow \infty, t \text { odd }} p_{t}(x)=\lim _{t \rightarrow \infty, t \text { even }} p_{t}(y)=\frac{1}{\gamma|G|}\left(\left(M+M_{1}-M_{2}\right)\left(M+M_{2}-M_{1}\right)^{\alpha}\right)^{\frac{1}{1+\alpha}}
\end{aligned}
$$

\footnotetext{
${ }^{12}$ The set $G_{1}$ must be a normal subgroup of $G$ with index 2 .
} 


\section{Monetary Shock II: Economic Effects}

This section is concerned with the effect of a monetary shock on economic variables in the fundamental equilibrium. The focus will be on (a) the behaviour of aggregate measures of both the price level and output, and (b) the behaviour of the distribution of individual prices and quantities across the economy.

The section begins by presenting simulations of the effects of a shock in the economies introduced in Examples 1 and 2 in Section 2. These provide a basic illustration of how prices and quantities respond. They also manifest certain regularities that help motivate the formal results that are presented in the remainder of the section. The first of these is that if $\alpha=2$, then a monetary shock always reduces aggregate output (Proposition 3). In other words, with a constant money stock, the economy achieves its global maximum for aggregate output in a steady state. An analogous result holds for prices: The average price level is below its steady-state value everywhere along its trajectory, as the economy converges to a steady state following a shock (Proposition 4). Although the proofs hold only for the case of $\alpha=2$, all of the simulations suggest that these results hold for a broader set of parameter values. The next set of results are based on a linear approximation of the equilibrium in the neighbourhood of the steady state; thus they indicate the approximate effects of a small monetary shock on both prices and quantities. Proposition 4 asserts that such a shock has, to a firstorder approximation, no effect on aggregate output. However, such a shock does alter the distribution of individual prices and quantities across the economy. Although the impact of the shock are, in principle, completely described by (10) and (11), in fact, these expressions are sufficiently complicated as to shed very little light on the observed distributional effects. As a consequence, Proposition 6 proposes an approximation to the quantities produced across the economy that is shown to be valid for all time periods beyond a sufficiently large $T$. Specifically, it asserts that a linear combination of a reasonably small set of eigenvectors belonging to $A$ provides a decent approximation to the distribution of individual quantities in all time periods $t>T$. Thus, where valid, this linear combination gives a nearly complete picture of the effects of a small monetary shock. This latter fact is complimented by Theorem 1 in Section 6, which indicates that these eigenvectors may, in many cases, be easily obtained, even for fairly large economies. Finally, a secondary theme running throughout the section is the tractability of the model: The closed-form solutions for the fundamental equilibrium, together with techniques in Section 6 for extracting eigenvalues and eigenvectors of $A$, allow the execution of precise 
numerical calculations, even in the case of some very large economies.

Two items of notation are required before getting underway. The expression $\frac{1}{|G|} \sum_{x \in G} p_{t}(x)$ is referred to as the average price level in period $t$, and is denoted by $P_{t}$, and $\sum_{x \in G} q_{t}(x)$ is referred to as the economy's real GDP in period $t$, and is abbreviated as GDP ${ }_{t}{ }^{13}$

The outcomes of two experiments are shown in Figures 3.a -3.d and 4.a - 4.d. In all cases, if the economy under consideration has $|G|$ vertices, then total quantity of cash is $1.1|G|$. In the initial distribution, $1+.1|G|$ units of currency are held at $e$, while 1 unit is held at every other location (in symbols, $m_{1}(e)=1+.1|G|$, and $m_{1}(x)=1$ for $x \neq e$ ). To motivate this choice for $m_{1}$, one can think of the economy as occupying its stationary steady state up until period 1, with one unit of currency being held at every vertex, but when period 1 arrives, cash balances equal to \%10 of the total money stock are transferred to the old agent at $e$. Thus, in this scenario, $m_{1}$ reflects an expansionary monetary policy. However, although it may be helpful to think in these terms, it is certainly not necessary. As the expressions in (10) and (11) make clear, the distribution of money prior to period $t$ has no effect whatsoever on prices or output from $t$ onwards. In other words, the following experiments are entirely consistent with any distribution of cash prior to period 1.

Figures 3.a - 3.d show the outcome of this experiment for an economy whose underlying graph is $C_{41}$ (the graph $C_{n}$ was introduced in Example 2.1). Figures 3.a and 3.b show the time series for $P_{t}$ and $G D P_{t}$, respectively. Figures 3.c show the distribution of prices across the economy in time periods $t=2,10,20,30,40,50$, and Figures 3.d show the distribution of output in the same time periods. The vertices are identified with the integers mod 40, with $e$ corresponding to 0 . The next set of figures describes the same experiment for an economy whose underlying graph is the hypercube $H_{40}$, which was introduced in Example 2.2. This graph has $2^{40}$ vertices. Figures 4.a and 4.b show the time series for $P_{t}$ and $G D P_{t}$, respectively, while the figures in 4.c and 4.d show the distribution of prices and quantities in periods $t=10,20,30,40,50,60$. To interpret these graphs, note that $H_{40}$ possesses the property of being distance transitive. As a consequence, vertices $x$ and $y$ are the same distance from $e$, then $p_{t}(x)=p_{t}(y)$ and $q_{t}(x)=q_{t}(y)$ for all $t$. In other words, agents that are the same distance from $e$ comprise an equivalence class. And

\footnotetext{
${ }^{13}$ If the base year is any period prior to the shock, and all prices are initially 1 , then $\sum_{x \in G} q_{t}(x)$ agrees with the economy's actual real GDP. (Of course this entails computing real GDP with respect to a base year, rather than by chain-weighting.) The variable $P_{t}$ corresponds to the economy's true consumer price index if, in addition to some mild assumptions, the representative consumption basket consists of all goods in the economy weighted equally. However, in the economies in this paper, no agent, in general, consumes anything even close to this representative basket; therefore $P_{t}$ does not really have the same interpretation as an actual consumer price index.
} 
altogether, there are $\left(\begin{array}{c}40 \\ d-1\end{array}\right)$ vertices that are a distance $d$ from $e$. In Figures 4.c and 4.d, a class of agents is identified with its distance from $e$. So, for instance, in periods 20 and 30 the quantity and price of the good produced by an agent who is 5 units from $e$ is indicated by a small triangle in the appropriate graphs.

Perhaps the most salient feature of a monetary shock is that any deviation from a steady state induced by such a shock entails a reduction GDP. ${ }^{14}$ Although I expect that this is true in general, I have been able to prove it only for the case where $\alpha=2$. Let $U \in \mathbb{R}^{|G|}$ be the uniform distribution on $G$; specifically, $U(x)=\frac{1}{|G|}$ for all $x \in G$. Recall that $\gamma|G|$ is the economy's steady state $G D P$.

Proposition 3: Let $\alpha=2$, and let $m_{1}$ be any initial distribution of money other than $U$. Then $G D P_{t}<\gamma|G|$ for all $t \geq 1$.

Figures 3.a and 4.a show a similar phenomenon for the price level. In particular a monetary shock induces an immediate jump in the price level, after which it remains always beneath its steady state value. As is the case for aggregate output, this is probably true in general, but I have obtained a proof only for the case where $\alpha=2$. Recall that in a steady state, the average price level is $\frac{M}{\gamma|G|}$.

Proposition 4: Let $\alpha=2$, and let $m_{1}$ be any initial distribution of money other than $U$. Then $P_{t}<\frac{M}{\gamma|G|}$ for all $t \geq 1$.

In the overlapping generations framework, where money is the only asset, a rising price level means that, on average, an additional unit of labour supplied by a young agent in period $t$ purchases a smaller quantity of consumption in period $t+1$. This implies that rising prices will, in general, reduce labour supply, and, hence, output. Moreover, in Figures 3.a and 4.a, the average price level increases monotonically over the course of the transition as the economy returns to its steady state. This, by itself will tend to reduce the economy's GDP. This helps to explain why a monetary shock causes a reduction in aggregate output. But it also just pushes the question back one step: Why does a monetary shock drive the average price level below its post-shock steady state value. Unfortunately, I do not have an answer to this question. A problem here is

\footnotetext{
${ }^{14}$ This fact is apparent in Figure 3.b, but is less so in Figure 4.b where GDP remains near its steady state level briefly before "dipping." The dip appears to be a feature of large economies. More specifically, it seems that the new cash transfers have to achieve a certain degree of dispersion relative to the size of the economy, before there is any noticeable impact on aggregate output.
} 
that the model does not, as far as I can see, allow money's role as a store of value to be disentangled from its role as a medium of exchange.

Another salient feature of the time series in Figures 3.b and 4.b is that the effect of the shock on aggregate output is surprisingly small. The largest deviation of GDP from its steady-state value in the economy on $C_{41}$ (which occurs in period 1) is only $0.36 \%$ (e.g. about $\frac{1}{3}$ of one percent), while the largest deviation for the economy $H_{40}$ (which occurs in period 14) is $.43 \%$. Although these magnitudes vary with both the size of the monetary shock and the value of the parameter $\alpha$, it seems that a very small deviation in aggregate output is a basic qualitative feature of the model. ${ }^{15}$

An important point that is evident from both equation (10) and Figures 3.c and 4.c, is that prices respond differentially to a monetary shock. In particular, it appears that prices at locations that are closer to the shock exhibit larger reactions than those that are further away. Also, prices appear to manifest stronger responses as the monetary disturbance draws closer. And, as been emphasized, this is the fundamental reason for the non-neutrality of money in the economy.

The next result makes the point that if the monetary shock is small then, to a firstorder approximation, aggregate output is not affected. Thus in the case of a small shock, all of the effects are on the distribution of output, and not on its level. The result is based on a linear approximation of the equilibrium in the neighbourhood of the steady state. Let $\phi_{0}, \phi_{1}, \ldots \phi_{|G|-1}$ be the eigenvectors of $A$, where $\phi_{0}=1_{G}$. Now any $m_{1}>0$ can be written as a function of $h=\left(h_{1}, \ldots, h_{|G|-1}\right) \in \mathbb{R}^{|G|-1}$ according to

$$
m_{1}\left(h_{1}, \ldots, h_{|G|-1}\right)=M \cdot 1_{G}+\sum_{i=1}^{|G|-1} h_{i} \phi_{i} .
$$

Note that if $h_{1}=h_{2}=\ldots=h_{|G|-1}=0$, then $m_{1}=M \cdot 1_{G}$, in which case the economy remains for all time in its steady state. Also, small values of $h_{i}$ correspond to a small monetary shock. So let $m_{1}$ be as in $(12)$, and let $q_{t}\left(x, h_{1}, \ldots, h_{|G|-1}\right)$ be the quantity produced at $x$ in period $t$, written so as to indicate its dependence on $\left(h_{1}, \ldots, h_{|G|-1}\right)$. Also, for any $x \in G$, let $\widehat{q}_{t}\left(x, h_{1}, \ldots, h_{|G|-1}\right)$ denote the first-order linear approximation of $q_{t}(x)$ at $\left(h_{1}, \ldots, h_{|G|-1}\right)=(0, \ldots, 0) .{ }^{16}$

\footnotetext{
${ }^{15} \mathrm{~A}$ doubling of the money supply produces a maximum deviation in GDP of $2.2 \%$ in the economy $C_{41}$, and maximum deviation of approximately $3.6 \%$ in the economy on $H_{40}$. If $\alpha=1.1$, then a $10 \%$ increase in the money supply generates a maximum deviation in $G D P$ of about $0.6 \%$ for $C_{41}$, and $2.1 \%$ for $H_{40}$.

${ }^{16}$ In other words, $\widehat{q}_{t}\left(x, h_{1}, \ldots, h_{|G|-1}\right)=q_{t}(x, 0, \ldots 0)+\sum_{i=1}^{|G|-1} \frac{\partial q_{t}(x, 0, \ldots, 0)}{\partial h_{i}} h_{i}$.
} 
Proposition 5: For any $h=\left(h_{1}, \ldots, h_{|G|-1}\right)$,

$$
\sum_{x \in G} \widehat{q}_{t}(x, h)=\gamma|G| .
$$

An analogous result holds for the average price level. Specifically, if $\widehat{p}_{t}(x, h)$ is the firstorder approximation to the price at $x$ in period $t$, where $h=\left(h_{1}, \ldots, h_{|G|-1}\right)$, then $\sum_{x \in G} \widehat{p}_{t}(x, h)=\frac{M}{\gamma}$.

The next result is an attempt at providing some means for understanding the distributional effect of a small monetary shock. It describes an approximation to the distribution of prices and quantities that is valid for all periods beyond a certain point in time. More specifically, it states that a linear combination of those eigenvectors that correspond to the eigenvalue of "second largest modulus," provides an approximation of the distribution of quantities in any period, where the approximation becomes more precise as $t$ increases. An analogous result holds for the distribution of prices. The result is not terribly deep: It is simply a manifestation of the fact that the rate at which the economy converges to its steady state is ultimately governed by the eigenvalue with largest modulus other than 1. Since all of the effects of a small monetary shock are on the distribution of output and prices (and not on their levels), this approximation, when valid, will give a nearly complete description of the effect of such a shock. The problem is that the time $T$ at which the approximation becomes valid may be too large for it to be useful. In particular, the minimum acceptable $T$ may be such that when $t>T$, the distributions of prices and quantities are essentially indistinguishable from their steady state distributions. Following are two examples, one where the approximation works extremely well, and one where it is essentially useless. In expect that the latter case is probably more typical.

Throughout the paper, the eigenvalues of $A$, including multiplicities, are denoted by $\lambda_{0}, \lambda_{1}, \ldots, \lambda_{|G|-1}$, and these are assumed to be labeled such that $1=\lambda_{0}>\lambda_{1} \geq$ $\ldots \geq \lambda_{|G|-1} \geq-1$. (See Lemma 0 in Section 7.) Let $\lambda_{S L E M}$ denote the "second largest eigenvalue modulus" for $A$. In other words, $\lambda_{S L E M}=\max \left\{\left|\lambda_{i}\right||| \lambda_{i} \mid<1\right\} .{ }^{17}$ Also, let $I^{+}$denote those indices for which the corresponding eigenvalues are both positive, and have modulus equal to $\lambda_{S L E M}$ (formally, $i \in I^{+}$if $0<\lambda_{i}<1$, and $\left|\lambda_{i}\right|=\lambda_{S L E M}$ ), and, similarly, let $I^{-}$be the set of indices corresponding to eigenvalues that are negative, and whose modulus is equal to $\lambda_{S L E M}$. It is possible for either $I^{+}$or $I^{-}$to be empty. Define the function $\widetilde{q}_{t}$ according to

$$
\widetilde{q}_{t}\left(x, h_{1}, \ldots, h_{|G|-1}\right)=\gamma+\gamma \cdot \lambda_{S L E M}^{t+1} \frac{1-\lambda_{S L E M}^{2}}{\alpha-\lambda_{S L E M}^{2}} \cdot \Theta(h, t)
$$

\footnotetext{
${ }^{17}$ The largest modulus among eigenvalues of $A$ is always 1 . The notation " $\lambda_{S L E M}$ " is borrowed from [?].
} 
where $\Theta(h, t)=\sum_{i \in I^{+}} h_{i} \phi_{i}(x)+(-1)^{t+1} \sum_{j \in I^{-}} h_{j} \phi_{j}(x)$. The definition of $\widetilde{p}_{t}$ is analogous, but with a minor modification to account for the possibility that $A$ can possess an eigenvalue equal to -1 :

$\widetilde{p}_{t}(x, h)= \begin{cases}\gamma^{-1}\left(\frac{M}{|G|}+\lambda_{S L E M}^{t+1} \frac{\alpha-1}{\alpha-\lambda_{S L E M}^{2}} \cdot \Theta(h, t)\right) & \text { if } \Gamma(G, \Omega) \text { is not bipartite } \\ \gamma^{-1}\left(\frac{M}{|G|}+\lambda_{S L E M}^{t+1} \frac{\alpha-1}{\alpha-\lambda_{S L E M}^{2}} \cdot \Theta(h, t)+(-1)^{t+1} h_{|G|-1} \phi_{|G|-1}(x)\right) & \text { if } \Gamma(G, \Omega) \text { is bipartite }\end{cases}$

where $h=\left(h_{1}, \ldots, h_{|G|-1}\right) \in \mathbb{R}^{|G|-1}$. It is important that these expressions are built from a potentially small number easily obtainable eigenvectors.

Proposition 6: There exist functions $\theta_{p}(h), \theta_{q}(h), \tau_{p}(h, t)$, and $\tau_{q}(h, t)$ such that

$$
\begin{aligned}
p_{t}(x, h) & =\widetilde{p}_{t}(x, h)+\theta_{p}(h)+\tau_{p}(h, t) \\
q_{t}(x, h) & =\widetilde{q}_{t}(x, h)+\theta_{q}(h)+\tau_{q}(h, t)
\end{aligned}
$$

where both $\theta_{p}=o(\|h\|)$ and $\theta_{q}=o(\|h\|)$ as $h \rightarrow 0$ and, for any fixed $h \in \mathbb{R}^{|G|-1}$, both $\tau_{p}(h, \cdot)=o\left(\lambda_{S L E M}^{t}\right)$ and $\tau_{q}(h, \cdot)=o\left(\lambda_{S L E M}^{t}\right)$ as $t \rightarrow \infty$.

Example 4.1: Here the economy is built on the graph $\Gamma\left(S_{10}, T_{10}\right)$, which has 10 ! = $3,628,800$ vertices, and the initial distribution of currency is as in the previous experiments: $m_{1}(e)=1+.1|G|$, and $m_{1}(x)=1$ if $x \neq e$. In this case the approximations $\widetilde{q}_{t}$, $t=1,2, \ldots$ take an especially simple form. The matrix $A$ has dimension $10 ! \times 10$ !, and therefore possesses 10 ! eigenvectors, so, in general, all 10 ! of these eigenvectors will appear in the sum (12). However in this particular case, $m_{1}$ is orthogonal to all but 42 eigenvectors, and of these, only two correspond to eigenvalues with modulus $\lambda_{S L E M}$. Therefore, the unpleasant looking sums $\Theta(h, t)$ contain only two terms. The precise expression for $\widetilde{q}_{t}$ is

$$
\widetilde{q}_{t}=\frac{1}{2}+\frac{81}{565}\left(\frac{7}{9}\right)^{t+1}\left(\phi_{1}+(-1)^{t+1} \phi_{|G|-2}\right)
$$

where $\phi_{1}$ and $\phi_{|G|-2}$ are eigenvectors corresponding to the eigenvalues $\frac{7}{9}$ and $-\frac{7}{9}$, respectively. As explained in Section 7, $\phi_{1}$ and $\phi_{|G|-1}$ can be read directly from the character table of $S_{10}$. Figure 5 shows the actual distribution of quantities across the economy, $q_{t}$, together with the approximation, $\widetilde{q}_{t}$, for $t=5,7,10$. To interpret the figure, note all agents within a conjugacy class are equivalent in all periods in terms of both the prices for their goods and the quantities that they produce. (In symbols, if $y=g^{-1} x g$ for some $g \in S_{n}$, then $q_{t}(x)=q_{t}(y)$ and $p_{t}(x)=p_{t}(y)$ for all $t$.) Also, there are 42 conjugacy classes in $S_{10}$. Therefore, although there are 10! vertices, in any time period 
there are only 42 distinct quantities and 42 distinct prices in the economy. ${ }^{18}$ Thus Figure 5 shows $q_{t}(x)$ and $\widetilde{q}_{t}(x)$, for one $x$ in each of the conjugacy classes of $S_{10}$. There is a comfortable gap between $\lambda_{S L E M}=\frac{7}{9}$ and the third largest eigenvalue modulus, which is $\frac{3}{5}$, so the approximation is quite accurate after about 7 periods. In this case, even though the economy has over 3 million sites, this simple approximation gives a nearly complete description of the effects of a small monetary shock for all $t \geq 7$.

Example 4.2: This second example features an economy built on $C_{101}$, the cyclic graph with 101 vertices, where the monetary shock is as in the previous examples. In this case, it takes sufficiently long for the term $\tau_{q}$ to shrink relative to $\widetilde{q}_{t}(x, h)$, that the approximation itself is indistinguishable from its long run equilibrium value. For instance, it takes 4,123 periods for $\frac{\left\|\tau_{q}\right\|}{\left\|\widetilde{q}_{t}(x, h)\right\|}$ to fall below .01, by which time $\widetilde{q}_{t}(x, h)$ has shrunk to the point that $\left\|\widetilde{q}_{t}(x, h)-\gamma \cdot 1_{G}\right\|<.00013 .{ }^{19}$ This is due to the fact that $C_{101}$ has a preponderance of eigenvalues whose moduli are very close to one. (Specifically, it has eight eigenvalues each of whose modulus is between .99 and 1, and two whose modulus is between .9995 and one.0

\section{Graph Properties and Convergence}

This section presents four examples that demonstrate that the rate at which an economy returns to a steady state following a monetary shock depends importantly on its spatial structure. In particular, it will be seen that different economies will manifest radically different rates of convergence due to differences in the structure of their underlying graphs. Although the relationship between the structure of the graph and the rates of convergence is not investigated systematically, it appears clear that the speed with which an economy re-equilibrates in the wake of a monetary shock is closely tied to the degree of "connectedness" of the underlying graph. ${ }^{20}$

The question of how rapidly an economy returns to its steady state following a monetary shock is clearly of fundamental importance. It seems intutively plausible that an economy that equilibrates more rapidly in the wake of a monetary shock will, for this reason alone, be inherently more stable. In particular, it would seem that if the structure of

\footnotetext{
${ }^{18}$ As explained in Section 7 , this is due to reasons: 1 ). the $m_{1}$ is a class function (that is, it is constant on conjugacy classes), and 2). Assumption G4. Indeed, this is one of the main reasons for adopting $G 4$.

${ }^{19}$ Recall that $\gamma \cdot 1_{G} \in \mathbb{R}^{|G|}$ is the steady state distribution of quantities.

${ }^{20}$ This is not too surprising as there exist deep theorems that connect the eigenvalues of the graph to measures of its connectedness such as its diameter, or its expanding constant. Many of these can be found in [12].
} 
an economy is such that the effects of a monetary shock dissipate rapidly, then monetary factors would be less imprtant as causes of bsusiness cycle fluctuations. This fact is of particular relevance to the recent history of the U.S. economy, as it seems clear that the volatility of business cycles has moderated over the past twenty-five years. The examples presented in this section suggest that an economy that manifests a greater degree of connectedness among its markets will undergo a less protracted fluctuation in response to a monetary shock. This in turn suggests that one factor that may contribute the reduced severity of U.S. business cycles, is a tendency for different sectors of the economy to be come increasingly inter-connected.

As indicated at the close of Subsection 2.3, the distribution of money can be regarded as evolving over time according to a Markov process. Thus the issue being investigated in this section is tantamount to examining the rate of convergence of a Markov processs on a finite group. This is a topic on which there exists an extensive literature ${ }^{21}$; in fact, it is sufficiently vast that there is no hope of even scratching its surface. Instead, the intention here is to present a small number of examples that demonstrate the broad range of behavior that is possible.

\subsection{The Rate of Convergence}

The section begins with a result that relates an economy's rate of convergence (to a steady state) to the eigenvalues of the underlying graph. This is especially convenient for reasons that will be described once the result is in place.

Fix a Cayley graph $\Gamma(G, \Omega)$ with adjacency matrix $A_{\Gamma}$ and diffusion matrix $A$. Recall that $u \in \mathbb{R}^{|G|}$ is the uniform distribution on $G$. Recall also that $A$ has an eigenvalue -1 if and only if $\Gamma(G, \Omega)$ is bipartite. In the event that $\Gamma(G, \Omega)$ is bipartite, let $G_{1}$ and $G_{2}$ denote the subsets of vertices as described prior to the Corollary of Section 4, and let $W_{G_{i}}$ be the function that takes the value $\frac{2}{|G|}$ on $G_{i}$, and zero on $G_{j}, j \neq i$. The set of complex-valued functions on $G$ is $\mathbb{C}^{|G|}$, the inner product on $\mathbb{C}^{|G|}$ is ${ }^{22}$

$$
\langle f, g\rangle=\frac{1}{|G|} \sum_{x \in G} f(x) \overline{g(x)},
$$

\footnotetext{
${ }^{21} \mathrm{~A}$ nice survey is provided by Coste-Saloffe in [24].

${ }^{22}$ All functions in this section are real-valued; however, complex-valued functions arise in the following section.
} 
and $\|\cdot\|$ is the norm $\|f\|=\langle f, f\rangle^{\frac{1}{2}}$. For fixed $x \in G, A^{t}(x, \cdot) \in \mathbb{R}^{|G|}$ represents the function $\left\{A^{t}(x, y) \mid y \in G\right\} .^{23}$

If $\Gamma(G, \Omega)$ is not bipartite, then $\left\|A^{t}(x, \cdot)-u\right\| \rightarrow 0$ as $t \rightarrow \infty$, while if $\Gamma(G, \Omega)$ is bipartite, then, for $x \in G_{i},\left\|A^{t}(x, \cdot)-W_{G_{i}}\right\| \rightarrow 0$ as $t \rightarrow \infty$. An economy whose underlying graph is non-bipartite (respectively, bipartite) converges to its steady state as $\left\|A^{t}(x, \cdot)-u\right\|$ (respectively. $\left.\left\|A^{t}(x, \cdot)-W_{G_{i}}\right\|\right)$ converges to 0 . Therefore, rates of convergence will be described in terms of the behavior of $\left\|A^{t}(x, \cdot)-u\right\|$ in the bipartite case, and $\left\|A^{t}(x, \cdot)-W_{G_{i}}\right\|$ in the non-bipartite case. Finally, note that if $\Gamma$ is any non-bipartite vertex transitive graph, the value of $\left\|A^{t}(x, \cdot)-u\right\|$ does not depend on $x$; likewise, if the graph is bipartite, then the number $\left\|A^{t}(x, \cdot)-W_{G_{i}}\right\|$ will not change if $x$ is replaced with any other member of $G_{i}$.

Proposition \%: If $\Gamma(G, \Omega)$ is non-bipartite, then for any $r \in G$

$$
\left\|A^{t}(r, \cdot)-u(\cdot)\right\|^{2}=\frac{1}{|G|^{2}} \sum_{j=1}^{|G|-1} \lambda_{j}^{2 t},
$$

whereas if $\Gamma(G, \Omega)$ is bipartite, then for any $r \in G_{i}$

$$
\left\|A^{t}(r, \cdot)-W_{G_{i}}(\cdot)\right\|^{2}=\frac{1}{|G|^{2}} \sum_{j=1}^{|G|-2} \lambda_{j}^{2 t} .
$$

The proposition describes the norms $\left\|A^{t}(r, \cdot)-u(\cdot)\right\|$ and $\left\|A^{t}(r, \cdot)-W_{G_{i}}(\cdot)\right\|$ in terms of the eigenvalues of $A$. There are three advantages to this formulation. It will be seen in the following section that the eigenvalues are often easy to obtain, even in cases where the graph is truly huge. Thus, the first reason is that these norms frame the question of convergence in terms of variables that are (often) readily available. The second reason is that the norms describe the rate of convergence in terms that do not depend on the particular choice of $m_{1}$. In other words, the rates of convergence depend only on the structure of the graph itself, and not on the potentially idiosyncratic aspects of the monetary shock. And third, the proposition gives exact expressions for the norms rather than merely an upper bound as is typical for results of this type.

\section{$5.2 \quad$ Examples}

Examples 5.1 - 5.3 present special cases of the cycle graph, $C_{n}$, the hypercube, $H_{n}$, and the transposition graph, $\Gamma\left(S_{n}, T_{n}\right)$, that were introduced in Subsection 2.1. In order for

\footnotetext{
${ }^{23}$ Remember that $A^{t}(x, y)$ is the element of the matrix $A^{t}$ whose row is indexed by $x \in G$, and whose column is indexed by $y \in G$.
} 
the examples to be comparable, the graphs were chosen to have approximately the same number of vertices. ${ }^{24} \mathrm{~A}$ fourth example introduces a special graph for which convergence is nearly instantaneous. It will be seen that the distribution of eigenvalues differs dramatically across the four cases, and, as a consequence, they manifest drastically different rates of convergence. It seems that the speed of convergence in these examples is much greater in graphs with higher elementary measures of connectedness, such as higher edge density, or smaller diameter.

Example 5.1: Here we consider the cycle graph on 5001 vertices. This is a non-bipartite graph with 5001 vertices, 5001 edges and a diameter of 2500. The 5001 eigenvalues of the corresponding adjacency matrix are $\cos \left(\frac{0}{5001} 2 \pi\right), \cos \left(\frac{1}{5001} 2 \pi\right), \ldots, \cos \left(\frac{5000}{5001} 2 \pi\right)$. These are obtained easily by taking the Fourier transform of the appropriate character, as described in the next section. ${ }^{25}$ The distance $\left\|A^{t}(x, \cdot)-u\right\|$ is shown for 600 periods (e.g. $t=1,2, \ldots 600)$ in Figure 6.a. The series is normalized so as to take the value 1 in period 1. It can be seen that convergence to the steady state is extremely slow; moreover, it appears to grow progressively slower as time unfolds. The reason can be seen clearly from histogram in Figure 7 which shows the distribution of the eigenvalues of the graph. Evidently there is a preponderance of eigenvalues whose modulus is very close to 1 . In particular, there are 2 eigenvalues equal to .999999211 and another 2 equal to -.999999803 . Altogether there are 44 eigenvalues whose modulus is between .9999 and 1.

Example 5.2: Here the graph is the hypercube, $H_{12}$, which is a bipartite graph with $2^{12}=$ 4096 vertices, 24576 edges, and a diameter of 12 . Each vertex has 12 neighbours. The eigenvalues together with their multiplicities are reported in Table 1. (Once again, these are found by elementary methods of Fourier analysis.) The value of $\left\|A^{t}(x, \cdot)-W_{G_{i}}\right\|$ for periods $t=1,2, \ldots, 20$, is shown in Figure 6.b. (normalized so that $\left\|A^{t}(x, \cdot)-W_{G_{i}}\right\|=1$ in period 1). Convergence is evidently very rapid: $\sqrt{\sum_{i=1}^{4095} \lambda_{i}^{2 t}}$ equals .1278 in period 20, and .0206 in period 30 .

Example 5.3: The graph here is $\Gamma\left(S_{7}, T_{7}\right)$, which is a bipartite graph possessing $7 !=5040$ vertices, 52920 edges, and a diameter of 6 . Each vertex has 21 neighbours. The eigenvalues and their multiplicities are shown in Table 2. These are obtained from the character

\footnotetext{
${ }^{24}$ The graph $C_{n}$ has $n$ vertices, $H_{n}$ has $2^{n}$ vertices, and $\Gamma\left(S_{n}, T_{n}\right)$ has $n$ ! vertices. Therefore, by setting $n=5001$ for $C_{n}, n=12$ for $H_{n}$, and $n=7$ for $\Gamma\left(S_{n}, T_{n}\right)$, all three have approximately 5000 vertices.

${ }^{25}$ Equivalently, the eigenvalues and their multiplicities can be found as application of Theorem 1 in the following section.
} 
table of $S_{7}$, as described in the following section. Since this graph has approximately twice the number of edges per vertex and half the diameter of $H_{12}$, one would expect convergence to occur more rapidly for this graph than for $H_{12}$, and indeed, Figure 6.b shows that this is the case. By period 20, $\sqrt{\sum_{i=1}^{5039} \lambda_{i}^{2 t}}=.00255$, and in period 30, $\sqrt{\sum_{i=1}^{5039} \lambda_{i}^{2 t}}<4 \times 10^{-5}$.

Example 5.4: Figure 8.a shows a Paley graph on 17 vertices. A Paley graph is Cayley graph where $|G|$ is a prime power satisfying $|G| \equiv 1 \bmod 4$, and where $\Omega$ consists of all non-zero squares mod $|G|$. (In other words, $\Omega$ is the set of quadratic residues in $\mathbb{Z}_{|G|}$.) Paley graphs are a special category of strongly regular graphs. A graph is strongly regular with parameters $(n, k, a, c)$ if the graph has $n$ vertices, if each vertex has $k$ neighbours, if every pair of adjacent vertices has a common neighbours, and if every pair of nonadjacent vertices has $c$ common neighbours. For example, the Petersen graph, shown in Figure 8.b, which is perhaps the most celebrated of all graphs, ${ }^{26}$ is strongly regular with $(n, k, a, c)=(10,3,0,1)$. A Paley graph with $q$ vertices is strongly regular with $(n, k, a, c)=\left(q, \frac{q-1}{2}, \frac{q-5}{4}, \frac{q-1}{4}\right)$. A strongly regular graph for which $c \geq 1$ is connected, in which case, its diameter is 2 . A Paley graph with $q$ vertices has eigenvalues and corresponding multiplicities as shown in Table 3. For such a graph

$$
|G|^{2}\left\|A^{t}(r, \cdot)-u\right\|^{2}=\frac{q-1}{2}\left(\frac{1}{(\sqrt{q}-1)^{2 t}}+\frac{1}{(\sqrt{q}+1)^{2 t}}\right)
$$

which shows that even for modest values of $q$, an economy on such a graph would converge to equilibrium almost instantaneously. For example, for $q=5009$ (a number chosen so as be comparable to the previous examples), $|G|^{2}\left\|A^{t}(r, \cdot)-u\right\|<10^{-11}$ by $t=4$.

Remark: Tables 1 - 3 show that the multiplicities of the eigenvalues for the graphs in examples $5.2-5.4$ are strikingly large. This is due to the very high degree of symmetry possessed by the respective graphs. To see this, fix a graph $\Gamma(V, E)$, not necessarily Cayley, with vertices $V$, edges $E$, and adjacency matrix $A_{\Gamma}$. To any graph automorphism $\pi: V \rightarrow V$ there corresponds a $|V| \times|V|$ permutation matrix $P$, obtained by setting $P(i, j)=1$ if $\pi\left(v_{i}\right)=v_{j}$, and setting $P(i, j)=0$ otherwise. If $\phi$ is an eigenvector of $A_{\Gamma}$ with eigenvalue $\lambda$, then $P \phi$ is also an eigenvector of $A_{\Gamma}$ with eigenvalue $\lambda$. (See [7], page 116.) In other words, the existence of an automorphism with permutation matrix $P$, gives rise to an eigenvector $P \phi$ sharing the same eigenvalue as $\phi$. And since highly

\footnotetext{
${ }^{26}$ In referring to the Petersen graph in [3], Cameron states: "This graph has far too many remarkable properties for even a brief survey here." (Page 204.)
} 
symmetric graphs possess large numbers of automorphisms, it can be expected that such graphs will possess eigenvalues with large multiplicities.

\section{Eigenvalues and Eigenvectors of $A$}

As the previous two sections make clear, the analysis of the fundamental equilibrium of the economies introduced in the paper is greatly facilitated if the eigenvalues and eigenvectors of $A$ (equivalently, $A_{\Gamma}$ ) can be obtained. This section shows that under the following conditions, the eigenvalues and eigenvectors are readily available: 1$)$. the underlying graph is a Cayley graph, 2). the generating set, $\Omega$, for the Cayley graph is the union of conjugacy classes, and 3 ). the initial distribution of money, $m_{1}$, is a class function. Under these conditions, the eigenvalues and eigenvectors of $A_{\Gamma}$ can be obtained directly from the character table of the underlying group. As is demonstrated throughout the paper, the implication is that very large and, ostensibly, complex economies can be investigated in great detail.

I begin by describing the importance of conditions (2) and (3) appearing in the previous paragraph. Once these basic facts are established, the concept of a character table is finally introduced. This will provide a sufficient basis to state the main theorem of the section. Two illustrative examples, one featuring an abelian group, and one featuring a non-abelian group, follow.

Let $G$ be a group with identity element $e$, and suppose that $G$ possesses $r$ conjugacy classes, denoted as $C_{1}, \ldots, C_{r}$. A function $f: G \rightarrow \mathbb{C}$ is a class function if it is constant on conjugacy classes; that is, if $f(x)=f(y)$ whenever $x$ and $y$ are conjugate to one another. The symbol $\mathcal{C}_{\mathcal{G}}$ will denote the set of class functions for $G$. There are two important facts regarding class functions: The first is that if $f$ and $g$ are class functions, then so is $f * g$, and the second is that if $\Omega$ is the union of conjugacy classes, then $\delta_{\Omega}$ is also a class function. ${ }^{27}$ Since $m_{t+1}=m_{1} * \delta_{\Omega}^{t}$, an immediate consequence is that if $m_{1}$ is a class function as well, then $m_{t}$ is a class function for all $t$. In other words, under condition (1) - (3) of the previous paragraph, the distribution of money in all time periods, $m_{t}$, is a class function.

Let $G L_{d}$ be the group of invertible $d \times d$ complex matrices, whose group operation is matrix multiplication. A matrix representation of $G$ is group homomorphism $\rho: G \rightarrow$ $G L_{d}$; specifically, $\rho(e)=I_{d}$, and $\rho(x y)=\rho(x) \rho(y)$, where $I_{d} \in G L_{d}$ is the identity matrix.

\footnotetext{
${ }^{27}$ The convolution product, $*$, and the function $\delta_{\Omega}$ were introduced in Section 2.3.
} 
Given a representation $\rho$ of $G$, a character of $\rho$ is a function $\chi: G \rightarrow \mathbb{C}$ defined according to $\rho(g)=\operatorname{tr} \rho(g)$, where, if $B \in G L_{d}$, then $\operatorname{tr} B$ is the trace of $B$. There exist $r$ characters of $G$ with the special property (defined in the following section) of being irreducible. These are fundamental in that all characters of a group are weighted sums of the irreducible characters. All characters of $G$ are class functions, and, consequently, each takes on at most $r$ distinct values (since there are assumed to be $r$ conjugacy classes). In fact, much more than this is true: the irreducible characters form an orthonormal basis for the set of class functions. The character table of $G$ is an $r \times r$ array that provides a complete list of the irreducible characters belonging to the group. Typically, the columns are indexed by the conjugacy classes, and the rows are indexed by the irreducible characters. So, for instance, if $\chi_{i}$ is the $i^{\text {th }}$ irreducible character, and $x \in G$ belongs to the $j^{\text {th }}$ conjugacy class, then $\chi_{i}(x)$ is the number appearing in row $i$ and column $j$ of the character table.

Example 6.1: The character table for the cyclic group of order 8 appears in Table 4 . The group is identified with $\mathbb{Z}_{8}=\{0,1, \ldots, 7\}$, and where, for simplicity, $\omega$ is written in place of $e^{\frac{1}{8} 2 \pi i}$. Since each element of an abelian group comprises its own conjugacy class, the conjugacy classes of $\mathbb{Z}_{8}$ are $0,1, \ldots, 7$; these are the column headings.

Example 6.2: The character table for $S_{7}$ appears in Figure 5.a. Each conjugacy class of $S_{n}$ corresponds to a partition of the integer $n .^{28}$ Therefore, each of the labels $C i$ across the top of the table corresponds to one of the 15 partitions of 7 . The partition corresponding to a specific $\mathrm{Ci}$, as well as the size of the given conjugacy class, is shown in Table 5.b.

The main theorem of the section can now be stated. It asserts that the irreducible characters of $G$ are eigenvectors for $A$, and that the corresponding eigenvalues are also comprised of ingredients found in the character table. Recall that for any $v \in \mathbb{C}^{|G|}, A v$ and $v * \delta_{\Omega}$ are equivalent.

Theorem: Let $\Gamma(G, \Omega)$ be a Cayley graph with underlying group $G$ and generating set $\Omega$, and let $\Omega$ satisfy Assumptions $G 0-G 4$. In particular, let $C_{1}, \ldots, C_{r}$ be conjugacy classes of $G$ such that $\Omega=C_{1} \cup \cdots \cup C_{r}$. If $\chi$ is any irreducible character of $G$, then

$$
\chi * \delta_{\Omega}=\lambda \cdot \chi
$$

\footnotetext{
${ }^{28}$ The partition of integer $n$ is a representation of $n$ as a sum of positive integer where order is unimportant.
} 
where $\lambda$ is a real number given by

$$
\frac{1}{|\Omega| \cdot \chi(e)}\left(\left|C_{1}\right| \chi\left(x_{1}\right)+\left|C_{2}\right| \chi\left(x_{2}\right)+\ldots+\left|C_{r}\right| \chi\left(x_{r}\right)\right) .
$$

where $x_{j}$ is any member of $C_{j}, j=1, \ldots, r$.

Finally, as described above, if $m_{1}$ is a class function, then the irreducible characters are only relevant eigenvectors of $A$. In other words, if $m_{1}$ is a class function, and if the graph adheres to Assumptions $G 0-G 4$, then the character table gives the full set of eigenvectors and eigenvalues for $A$. So for instance, the expressions in (10) and (11) are built from terms of the form $A^{j} m_{t}$, which in turn, is just $m_{t+j}$. Now if $m_{1}$ is class function, then, for all $t, m_{t+1}=\sum_{i}\left\langle m_{1}, \chi_{i}\right\rangle \lambda_{i}^{t} \chi_{i}$, where the sum is over the irreducible characters of $G$.

Example 1: Consider the economy whose spatial structure is given by the Cayley graph constructed on the group $\mathbb{Z}_{8}$ with generating set $\Omega=\{-1,1\}$, ( $\left.\bmod 8\right)$. The eight eigenvectors of $\delta_{\Omega}$ are the eight rows of the character table for $\mathbb{Z}_{8}$, and the corresponding eigenvalues are

$$
\frac{1}{2}\left(\overline{\chi_{j}(1)}+\overline{\chi_{j}(-1)}\right)=\frac{1}{2}\left(e^{-\frac{j}{8} 2 \pi i}+e^{\frac{j}{8} 2 \pi i}\right)=\cos (j / 8 \cdot 2 \pi)
$$

for $j=0,1 \ldots, 7{ }^{29}$

Example 2: Let $S_{7}$ be the underlying group, and let $T_{7}$ be the generating set. The set $T_{7}$ is itself a conjugacy class. Therefore, according to the Theorem above, if $\chi$ is an irreducible character, then the corresponding eigenvalue is $\frac{\chi(\tau)}{\chi(e)}$, where $\tau$ is any element in $T_{7}$. The terms in the $\chi(e)$ comprise the first column of the character table, while the terms $\chi(\tau)$ comprise the second column. The multiplicity of an eigenvalue $\frac{\chi(\tau)}{\chi(e)}$ is $\chi(e)^{2}$. All of the entries in Table 2 are obtained in this fashion. For instance, the entries in columns 2 and 13 of Table 2 correspond to characters $\chi_{2}$ and $\chi_{13}$ in Table 5.a. Thus the eigenvalues are $\frac{\chi_{2}(\tau)}{\chi_{2}(e)}=\frac{4}{6}$ and $\frac{\chi_{13}(\tau)}{\chi_{13}(e)}=\frac{-6}{14}$, and their multiplicities are $\chi_{2}(e)^{2}=6^{2}$ and $\chi_{13}(e)^{2}=14^{2}$.

Remark 1: Note that in the special case where $G$ is an abelian group, Theorem 1 is obtained by taking the Fourier transform of the function $\delta_{\Omega}$. To see this, let $G$ be a finite

\footnotetext{
${ }^{29}$ Note that although the eigenvectors are complex, the real and complex components taken separately give real eigenvectors. That is, if $u_{j}+i v_{j}, j=0, \ldots, 7$, is one of the rows of the character table, then both $\left(u_{0}, u_{1}, \ldots, u_{7}\right)$ and $\left(v_{0}, v_{1}, \ldots, v_{7}\right)$ are real eigenvectors.
} 
abelian group, and let $\widehat{G}$ be the characters of $G$. (The characters of an abelian group $G$ are the homomorphisms from $G$ into the unit circle in $\mathbb{C}$.) For a function $f: G \rightarrow \mathbb{C}$, the Fourier transform of $f$ is the function $\widehat{f}: \widehat{G} \rightarrow \mathbb{C}$ defined according to

$$
\widehat{f}(\chi)=\sum_{x \in G} f(x) \overline{\chi(x)}
$$

Therefore, for any $f \in \mathbb{C}^{|G|}$, and any $\chi \in \widehat{G}$,

$$
\begin{aligned}
\chi * f(x) & =\sum_{y \in G} \chi\left(x y^{-1}\right) f(y) \\
& =\sum_{y \in G} \chi(x) \chi\left(y^{-1}\right) f(y) \\
& =\left(\sum_{y \in G} f(y) \overline{\chi(y)}\right) \chi(x) \\
& =\widehat{f}(\chi) \chi(x),
\end{aligned}
$$

Now if $f$ is replaced with $\delta_{\Omega}$, this becomes $\chi * \delta_{\Omega}(x)=\widehat{\delta_{\Omega}}(\chi) \chi(x)$. In other words, the character $\chi$ is an eigenvector of $\delta_{\Omega}$ whose corresponding eigenvalue is the Fourier transform $\widehat{\delta_{\Omega}}$ evaluated at $\chi$; that is $\widehat{\delta_{\Omega}}(\chi) \in \mathbb{C}$.

Although these ideas can be generalized to the setting of non-abelian groups, the generalization is so extensive that it no longer really looks like Fourier analysis. Consequently, Theorem 1 is expressed here in the language of representations of groups. ${ }^{30}$

Remark 2: The fact that the character table gives a complete list of the eigenvalues of $A$ is independent of whether the functions $m_{t}, t=1,2, \ldots$, are class functions. The theorem above gives a complete description of the eigenvalues. Their multiplicities can be found from the first column of the character table: If $\chi$ is an irreducible character, then the multiplicity of the corresponding eigenvalue is $\chi(e)^{2}$. So, for instance, the eigenvalue corresponding to $\chi_{3}$ is $\frac{6}{14}$ (as described in Example 2 above), and its multiplicity is $14^{2}$. Thus the table following Example BLANK, which contains a full set of eigenvalues together with their multiplicities, is obtained by reading down the first two columns of the character table.

\footnotetext{
${ }^{30}$ For an excellent demonstration of why the calculation in Remark 1 fails if the group is non-abelian, see Chapter 103 of [21]. A proof of the Theorem in this section can be found at BLANK. It approaches the result from the perspective of the theory representation of finite groups. Diaconnis develops the Theorem in BLANK by approaching it from the perspective of generalized Fourier Analysis.
} 


\section{Proofs}

The following lemma gathers together some basic facts concerning the diffusion matrix $A$ corresponding to a connected Cayley graph.

Lemma 0: Let $A_{\Gamma}$ and $A$ be the adjacency and diffusion matrices, respectively, for a connected Cayley graph $\Gamma(G, \Omega)$, and let $\lambda_{0}, \ldots, \lambda_{|G|-1}$ be the eigenvalues of $A$, including multiplicities, whose corresponding eigenvectors are $\phi_{0}, \ldots, \phi_{|G|-1}$.

1. $A$ is symmetric

2. The eigenvalues of $A$ are real and can be labeled such that

$$
1=\lambda_{0}>\lambda_{1} \geq \cdots \geq \lambda_{|G|-1} \geq-1 \text {. }
$$

3. $A 1_{G}=1_{G}$ (that is, $\left.\phi_{0}=1_{G}\right)$, and $\sum_{x \in G} \phi_{i}(x)=0$ for all $i=1, \ldots,|G|-1$.

4. $\lambda_{|G|-1}=-1$ if and only if $\Gamma(G, \Omega)$ is bipartite.

Proof: All statements in the lemma follow from well-known facts concerning the adjacency matrix $A_{\Gamma}$; since $A$ and $A_{\Gamma}$ are related according to $A=\frac{1}{|\Omega|} A_{\Gamma}, A$ inherits the basic properties of $A_{\Gamma}$. That $A_{\Gamma}$ is symmetric follows from Assumption $G 2$; and since $A_{\Gamma}$ is symmetric, its eigenvalues are real, and its eigenvectors are mutually orthogonal. That the eigenvalues of $A_{\Gamma}$ are bounded in modulus by $|\Omega|$ is given in Proposition 3.1 of [7]. That $1_{G}$ is an eigenvector of $A_{\Gamma}$ with eigenvalue $|\Omega|$ follows from the fact that all vertices of a Cayley graph have the same number of neighbours. (In the language of graph theory, Cayley graphs are $|\Omega|$-regular.) Assumption $G 3$ guarantees that $\Gamma(G, \Omega)$ is connected, which, in turn, implies that the eigenvalue $|\Omega|$ has multiplicity 1 (Proposition 3.1 of [7]). That $\sum_{x \in G} \phi_{i}(x)=0$ for $i \neq 0$ follows from the fact that $\phi_{i}$ is orthogonal to $1_{G}$. The truth of the fourth point is contained in Section 2c of [7] (page 11).

Proof of Propositions 1 and 2: An equilibrium is characterized by sequences of positive functions

$$
p_{t}: G \rightarrow \mathbb{R}, \quad q_{t}: G \rightarrow \mathbb{R}, \quad m_{t}: G \rightarrow \mathbb{R}
$$


$t=1,2, \ldots$, satisfying

$$
\begin{aligned}
q_{t}(x) & =\gamma\left(\frac{p_{t}(x)}{\prod_{y \in N(x)} p_{t+1}(y)^{\frac{1}{\Omega \mid}}}\right)^{\frac{1}{\alpha-1}} \\
m_{t+1}(x) & =\frac{1}{|\Omega|} \sum_{y \in N(x)} m_{t}(y) \\
p_{t}(x) q_{t}(x) & =\frac{1}{|\Omega|} \sum_{y \in N(x)} m_{t}(y)
\end{aligned}
$$

Substitute (13) into (15), and then solve for $p_{t}(x)$ :

$$
p_{t}(x)=\alpha^{\frac{1}{\alpha}}\left(\frac{1}{\Omega \mid} \sum_{y \in N(x)} m_{t}(y)\right)^{1-\frac{1}{\alpha}}\left(\prod_{z \in N(x)} p_{t+1}(z)^{\frac{1}{\Omega \mid}}\right)^{\frac{1}{\alpha}} .
$$

This latter expression holds for each $x \in G$. Now take logarithms of both sides for each equation, and express the results in vector notation. (In other words, $\log p_{t} \in \mathbb{R}^{|G|}$.)

$$
\log p_{t}=\log \alpha^{\frac{1}{\alpha}} 1_{G}+\left(1-\frac{1}{\alpha}\right) \log A m_{t}+\frac{1}{\alpha} A \log p_{t+1}
$$

This is system of first-order difference equations that can be solved forward for $p_{t+s}$, $s>0$, starting from any $p_{t}$. Therefore, any choice of $p_{1}>0$ generates an sequence of (positive) equilibrium prices. This establishes part (1) of Proposition 1. Now iterating (17) forward yields

$$
\log p_{t}=\frac{\alpha-\frac{1}{\alpha^{s}}}{\alpha-1} \log \alpha^{\frac{1}{\alpha}} 1_{G}+\left(1-\frac{1}{\alpha}\right) \sum_{n=0}^{s} \frac{1}{\alpha^{n}} A^{n} \log A^{n} m_{t+1}+\frac{1}{\alpha^{s+1}} A^{s+1} \log p_{t+s+1}
$$

(The fact that $A 1_{G}=1_{G}$ was used here.) Taking the limit of the righthand side, and, in the process, using $\lim _{n \rightarrow \infty} \frac{1}{\alpha^{n+1}} A^{n+1} \log p_{t+n+1}=0$, leaves

$$
\log p_{t}=\log \alpha^{\frac{1}{\alpha-1}} 1_{G}+\left(1-\frac{1}{\alpha}\right) \sum_{s=0}^{\infty} \frac{1}{\alpha^{s}} A^{s} \log A^{s} m_{t+1} .
$$

The prices obtained here are clearly positive, and by Corollary BLANK, they are also bounded. Thus, this is the "fundamental equilibrium." This also takes care of Proposition 
2. Note that the condition $\lim _{n \rightarrow \infty} \frac{1}{\alpha^{n+1}} A^{n+1} \log p_{t+n+1}=0$ functions essentially as a transversality condition.

To prove part (3) of Proposition 1, assume that the condition $\lim _{n \rightarrow \infty} \frac{1}{\alpha^{n}} A^{n} \log p_{t+n}=$ 0 fails to hold; in particular, assume that there is a subsequence $\left\{n_{k}\right\}$ such that $\lim _{k \rightarrow \infty} \frac{1}{\alpha^{n_{k}}} B^{n_{k}} \log p_{t+n_{k}}=$ $c$, for some $c=\left(c_{1}, \ldots, c_{|G|}\right) \neq 0$. There are two cases to be considered: either $0<c_{j} \leq \infty$ for some $j$, or $-\infty \leq c \leq 0$. I start with the first of these. If there is a $j$ for which $0<c_{j}<\infty$, then there is a number $0<M<c_{j}$ and an integer $K_{1}$, such that if $k>K_{1}$ then $\frac{1}{\alpha^{n_{k}}} A^{n_{k}} \log p_{t+n_{k}}\left(x_{j}\right)>M$. This last inequality can be re-arranged to give

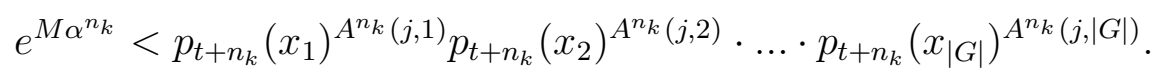

(Here $A^{n_{k}} r, s(k)$ is the entry in row $r$ and column $s$ of the matrix $A^{n_{k}}$.) An application of the AM-GM inequality yields

$$
e^{M \alpha^{n_{k}}}<A^{n_{k}}(j, 1) p_{t+n_{k}}\left(x_{1}\right)+A^{n_{k}}(j, 2) p_{t+n_{k}}\left(x_{2}\right)+\ldots+A^{n_{k}}(j,|G|) p_{t+n_{k}}\left(x_{|G|}\right) .
$$

Now since $A^{n_{k}}(r, s) \rightarrow \frac{1}{|G|}$ as $n_{k} \rightarrow \infty$ for all $r$ and $s$, it follows that there is a $K_{2}$ such that if $k>K_{2}$ then $A^{n_{k}}(r, s)<\frac{2}{|G|}$ for all $r, s$, and, therefore, if $k>\max \left\{K_{1}, K_{2}\right\}$, then

$$
\frac{1}{2} e^{M \alpha^{n_{k}}}<\frac{p_{t+n_{k}}\left(x_{1}\right)+p_{t+n_{k}}\left(x_{2}\right)+\ldots+p_{t+n_{k}}\left(x_{|G|}\right)}{|G|},
$$

which is the first inequality of part (a).

Now for the second case, assume that $-\infty \leq c_{j}<0$ for some $j$. In this case a sufficiently large $k$ ensures that both $\frac{1}{\alpha^{n_{k}}} A^{n_{k}} \log p_{t+n_{k}}<-M$ and $A^{n_{k}}(r, s)>\frac{1}{2|G|}$ for all $r, s$, where $M$ is a positive number satisfying $c_{j}<-M<0$. Then

$$
\begin{aligned}
& e^{-M \alpha^{n_{k}}}>p_{t+n_{k}}\left(x_{1}\right)^{A^{n_{k}(j, 1)}} p_{t+n_{k}}\left(x_{2}\right)^{A^{n_{k}(j, 2)}} \cdot \ldots \cdot p_{t+n_{k}}\left(x_{|G|}\right)^{A^{n_{k}(j,|G|)}} \\
& \geq \frac{1}{\frac{A^{n_{k}(j, 1)}}{p_{t+n_{k}}\left(x_{1}\right)}+\frac{A^{n_{k}(j, 2)}}{p_{t+n_{k}}\left(x_{2}\right)}+\cdots+\frac{A^{n_{k}(j,|G|)}}{p_{t+n_{k}}\left(x_{|G|}\right)}} \\
& >2|G| \frac{1}{\frac{1}{p_{t+n_{k}}\left(x_{1}\right)}+\frac{1}{p_{t+n_{k}}\left(x_{2}\right)}+\cdots+\frac{1}{p_{t+n_{k}}\left(x_{|G|}\right)}}
\end{aligned}
$$

where the transition to the second line employs the GM-HM inequality. Therefore, if $k$ is sufficiently large then

$$
2|G| e^{M \alpha^{n_{k}}}<\frac{1}{p_{t+n_{k}}\left(x_{1}\right)}+\frac{1}{p_{t+n_{k}}\left(x_{2}\right)}+\cdots+\frac{1}{p_{t+n_{k}}\left(x_{|G|}\right)},
$$


which is the first inequality of part (b).

I now generate the remaining two inequalities. Since $\lim _{t \rightarrow \infty} m_{t}(x)=\frac{1}{|G|}$ for all $x \in G$, it follows from (15) that $\lim _{t \rightarrow \infty} p_{t}(x) q_{t}(x)=\frac{1}{|G|}$ for all $x \in G$. Therefore, for any $\epsilon>0$ there is a $K$ such that $k>K$ implies

$$
\frac{1-\epsilon}{|G|}<p_{t+k}(x) q_{t+k}(x)<\frac{1+\epsilon}{|G|}
$$

for all $x \in G$. Therefore, $k>K$ also implies both

$$
p_{t+k}\left(x_{1}\right)+p_{t+k}\left(x_{2}\right)+\ldots+p_{t+k}\left(x_{|G|}\right)<\frac{(1+\epsilon)}{|G|}\left(\frac{1}{q_{t+k}\left(x_{1}\right)}+\frac{1}{q_{t+k}\left(x_{2}\right)}+\cdots+\frac{1}{q_{t+k}\left(x_{|G|}\right)}\right)
$$

and

$$
\frac{1}{p_{t+k}\left(x_{1}\right)}+\frac{1}{p_{t+k}\left(x_{2}\right)}+\cdots+\frac{1}{p_{t+k}\left(x_{|G|}\right)}<\frac{|G|}{(1-\epsilon)}\left(q_{t+k}\left(x_{1}\right)+q_{t+k}\left(x_{2}\right)+\ldots+q_{t+k}\left(x_{|G|}\right)\right)
$$

BLANK together with BLANK imply BLANK, while, BLANK and BLANK imply BLANK.

Proof of Proposition 3: It will help to introduce some simplifying notation. Let $D_{i}$ stand in for $m_{t+1}\left(x_{1}\right)^{A(i, 1)} m_{t+1}\left(x_{2}\right)^{A(i, 2)} \cdot \ldots \cdot m_{t+1}\left(x_{|G|}\right)^{A(i,|G|)}, i=1, \ldots,|G|$, where, as always, $A(i, j)$ is the element in row $i$ and column $j$ of $A$. Then equations (13)-(15) imply that $q_{t}\left(x_{i}\right)$ can be written as

$$
q_{t}\left(x_{i}\right)=\frac{1}{\alpha} \frac{1}{\alpha}\left(\frac{m_{t+1}\left(x_{i}\right)}{D_{i}}\right)^{\frac{1}{\alpha}}\left(q_{t+1}\left(x_{1}\right)^{A(i, 1)} q_{t+1}\left(x_{2}\right)^{A(i, 2)} \cdot \ldots \cdot q_{t+1}\left(x_{|G|}\right)^{A(i,|G|)}\right)^{\frac{1}{\alpha}}
$$

Therefore,

$G D P_{t}=\sum_{i=1}^{|G|} q_{t}\left(x_{i}\right)=\frac{1}{\alpha}{ }^{\frac{1}{\alpha}} \sum_{i=1}^{|G|}\left(\frac{m_{t+1}\left(x_{i}\right)}{D_{i}} \cdot q_{t+1}\left(x_{1}\right)^{A(i, 1)} q_{t+1}\left(x_{2}\right)^{A(i, 2)} \cdot \ldots \cdot q_{t+1}\left(x_{|G|}\right)^{A(i,|G|)}\right)^{\frac{1}{\alpha}}$

Next, specialize to the case of $\alpha=2$, and then apply the Cauchy-Schwartz inequality to obtain

$$
\sum_{i=1}^{|G|} q_{t}\left(x_{i}\right) \leq \frac{1}{\alpha}^{\frac{1}{\alpha}}\left(\sum_{i=1}^{|G|} \frac{m_{t+1}\left(x_{i}\right)}{D_{i}}\right)^{\frac{1}{2}}\left(\sum_{i=1}^{|G|} q_{t+1}\left(x_{1}\right)^{A(i, 1)} q_{t+1}\left(x_{2}\right)^{A(i, 2)} \cdot \ldots \cdot q_{t+1}\left(x_{|G|}\right)^{A(i,|G|)}\right)^{\frac{1}{2}} .
$$


Now set to work on the term $\sum_{i=1}^{|G|} \frac{m_{t+1}\left(x_{i}\right)}{D_{i}}$. Using the GM-HM inequality 31

$$
\begin{array}{r}
\frac{m_{i}\left(x_{t+1}\right)}{D_{i}} \leq \frac{A(i, 1) m_{t+1}\left(x_{i}\right)}{A(1,1) m_{t+1}\left(x_{1}\right)+\cdots+A(1,|G|) m_{t+1}\left(x_{|G|}\right)}+\frac{A(i, 2) m_{t+1}\left(x_{i}\right)}{A(2,1) m_{t+1}\left(x_{1}\right)+\cdots+A(2,|G|) m_{t+1}\left(x_{|G|}\right)}+\ldots \\
\cdots+\frac{A(i,|G|) m_{t+1}\left(x_{i}\right)}{A(|G|, 1) m_{t+1}\left(x_{1}\right)+\cdots+A(|G|,|G|) m_{t+1}\left(x_{|G|}\right)}
\end{array}
$$

where equality holds if and only if $m_{t+1}\left(x_{1}\right)=m_{t+1}\left(x_{2}\right)=\ldots=m_{t+1}\left(x_{|G|}\right)$. Since $A$ is symmetric, it follows that

$$
\begin{aligned}
\sum_{i=1}^{n} \frac{m_{t+1}\left(x_{i}\right)}{D_{i}} & \leq \sum_{i=1}^{|G|}\left(\frac{A(i, 1) m_{t+1}\left(x_{i}\right)}{A(1,1) m_{t+1}\left(x_{1}\right)+\cdots+A(1,|G|) m_{t+1}\left(x_{|G|}\right)}+\cdots+\frac{A(i,|G|) m_{t+1}\left(x_{i}\right)}{A(|G|, 1) m_{t+1}\left(x_{1}\right)+\cdots+A(|G|,|G|) m_{t+1}\left(x_{|G|}\right)}\right) \\
& =|G|
\end{aligned}
$$

Now focus on the term $\sum_{i=1}^{|G|}\left(q_{t+1}\left(x_{1}\right)^{A(i, 1)} q_{t+1}\left(x_{2}\right)^{A(i, 2)} \cdot \ldots \cdot q_{t+1}\left(x_{|G|}\right)^{A(i,|G|)}\right)$. Applying the AM-GM inequality gives

$$
\begin{aligned}
\sum_{i=1}^{|G|}\left(q_{t+1}\left(x_{1}\right)^{A(i, 1)} q_{t+1}\left(x_{2}\right)^{A(i, 2)} \cdot \ldots \cdot q_{t+1}\left(x_{|G|}\right)^{A(i,|G|)}\right) & \leq \sum_{i=1}^{|G|}\left(A(i, 1) q_{t+1}\left(x_{1}\right)+\cdots+A(i,|G|) q_{t+1}\left(x_{|G|}\right)\right) \\
& =q_{t+1}\left(x_{1}\right)+\cdots+q_{t+1}\left(x_{|G|}\right) \\
& =G D P_{t+1}
\end{aligned}
$$

where the transition to the second line is due to the fact that the columns of $A$ sum to 1. So putting this together causes (18) to go over to

$$
G D P_{t} \leq \frac{1}{\alpha} \frac{1}{\alpha}|G|^{\frac{1}{2}} G D P_{t+1}^{\frac{1}{2}}
$$

where, once again, the inequality is strict unless $m_{t+1}\left(x_{1}\right)=\ldots=m_{t+1}\left(x_{|G|}\right)$. However, (19) implies

$$
G D P_{t} \leq\left(\frac{1}{\alpha} \frac{1}{\alpha}|G|^{\frac{1}{2}}\right)^{\left(1+\frac{1}{2}+\frac{1}{2^{2}}+\ldots+\frac{1}{2^{n}}\right)} G D P_{t+n+1}^{\frac{1}{2^{n+1}}}
$$

Since $\lim _{t \rightarrow \infty} G D P_{t}=|G| \gamma$, it follows that $\lim _{n \rightarrow \infty} G D P_{t+n+1}^{\frac{1}{2^{n+1}}}=1$. Therefore (20) implies that $G D P_{t} \leq \frac{1}{\alpha}^{\frac{2}{\alpha}}|G|$. But then

$$
G D P_{t+1} \leq \frac{1}{\alpha}^{\frac{2}{\alpha}}|G|
$$

\footnotetext{
${ }^{31}$ Specifically, if $p_{1}+\cdots+p_{n}=1$, and if all terms are positive, then $\left(x_{1}^{p_{1}} x_{2}^{p_{2}} \cdot \ldots \cdot x_{n}^{p_{n}}\right)^{-1}<\frac{p_{1}}{x_{1}}+\cdots+\frac{p_{n}}{x_{n}}$.
} 
is true as well. But since (19) holds with equality only if and only if $m_{t}\left(x_{1}\right)=\ldots=$ $m_{t}\left(x_{|G|}\right)$, it follows that, together, (19) and (21) imply

$$
G D P_{t}<\frac{1}{\alpha} \frac{2}{\alpha}|G|
$$

provided that $m_{t+1}\left(x_{1}\right)=\ldots=m_{t+1}\left(x_{|G|}\right)$ does not hold. Finally, if $\alpha=2$, then $\alpha^{\frac{2}{\alpha}}=$ $\frac{1}{2}=\gamma$, which proves the result.

Proof of Proposition 4: The proof follows the same format as that of Proposition 5; however, it is much less complicated. The expression in (16) can be written as

$$
p_{t}\left(x_{i}\right)=\alpha^{\frac{1}{\alpha}}\left(\sum_{j=1}^{|G|} A(i, j) m_{t}\left(x_{j}\right)\right)^{1-\frac{1}{\alpha}}\left(p_{t+1}\left(x_{1}\right)^{A(i, 1)} p_{t+1}\left(x_{2}\right)^{A(i, 2)} \cdot \ldots \cdot p_{t+1}\left(x_{\mid G}\right)^{A(i,|G|)}\right)^{\frac{1}{\alpha}} .
$$

Summing over all $i$, and using the Cauchy Schwartz inequality gives

$$
\sum_{i=1}^{|G|} p_{t}\left(x_{i}\right) \leq \alpha^{\frac{1}{\alpha}}\left(\sum_{i=1}^{|G|}\left(\sum_{j=1}^{|G|} A(i, j) m_{t}\left(x_{j}\right)\right)^{2\left(1-\frac{1}{\alpha}\right)}\right)^{\frac{1}{2}}\left(\sum_{i=1}^{|G|}\left(p_{t+1}\left(x_{1}\right)^{A(i, 1)} \ldots \cdot p_{t+1}\left(x_{\mid G}\right)^{A(i,|G|)}\right)^{\frac{2}{\alpha}}\right)^{\frac{1}{2}}
$$

Specialize to $\alpha=2$, and then consider the righthand side of (22). The middle term, namely, is equal to $\sum_{i=1}^{|G|} m_{t}\left(x_{i}\right)$, which, in turn, is just $M$. Also, the contents of the parentheses in the third term is treated as follows

$$
\begin{aligned}
\sum_{i=1}^{|G|}\left(p_{t+1}\left(x_{1}\right)^{A(i, 1)} \cdot \ldots \cdot p_{t+1}\left(x_{|G|}\right)^{A(i,|G|)}\right)^{\frac{1}{\alpha}} & \leq \sum_{i=1}^{|G|}\left(A(i, 1) p_{t+1}\left(x_{1}\right)+\ldots+A(i,|G|) p_{t+1}\left(x_{|G|}\right)\right)^{\frac{1}{2}} \\
& =p_{t+1}\left(x_{1}\right)+\cdots+p_{t+1}\left(x_{|G|}\right),
\end{aligned}
$$

where the transition to the second uses the fact that the columns of $A$ sum to 1 . Also, the inequality here is strict as long as the $p_{t+1}(i), i=1, \ldots, n$ are not all equal. Substituting these changes into (22) produces

$$
\sum_{i=1}^{|G|} p_{t}\left(x_{i}\right) \leq \alpha^{\frac{1}{\alpha}} M^{\frac{1}{2}} \sum_{i=1}^{|G|} p_{t+1}\left(x_{i}\right)
$$

or, equivalently, $P_{t} \leq \alpha^{\frac{1}{\alpha}} M^{\frac{1}{2}} P_{t+1}$. Now proceed from here as in the last part of the proof of Proposition 5, in order to obtain $P_{t} \leq \frac{M}{\gamma}$, where the inequality is strict if the $p_{t+1}\left(x_{i}\right)$, $i=1, \ldots, n$ are not all equal. 
Proof of Proposition 5: Some pencil-work produces

$$
\widehat{p}_{t}(x, h)=\frac{M}{|G| \gamma}+\frac{1}{\gamma} \sum_{i=1}^{|G|-1} \lambda_{i}^{t} \frac{\alpha-1}{\alpha-\lambda_{i}^{2}} h_{i} \phi_{i}(x) \quad \text { and } \quad \widehat{q}_{t}(x, h)=\gamma+\gamma \sum_{i=1}^{|G|-1} \lambda_{i}^{t} \frac{1-\lambda_{i}^{2}}{\alpha-\lambda_{i}^{2}} h_{i} \phi_{i}(x)
$$

The result now follows by summing over all $x \in G$, since $\sum_{x \in G} \phi_{i}(x)=0$ for $i=$ $1, \ldots,|G|-1$.

Proof of Proposition 6: Simply set $\widehat{q}_{t}(x, h)=\widetilde{q}_{t}(x, h)+\tau_{h}(t) ; \widetilde{q}_{t}(x, h)$ is then obtained from $\widehat{q}_{t}(x, h)$ by discarding those terms in the latter sum for which $\left|\lambda_{i}\right|<\lambda_{S L E M}$.

Proposition 7: Saloffe-Coste indicates how to obtain Proposition 8 for the non-bipartite case by means of an eigenvector decomposition of $A$ (see [24], Theorem 5.2). However, a shorter proof is presented here. It is based on the equality (23), which is proved in [1] (page 26 of Chapter 3):

$$
\sum_{j=0}^{|G|-1}\left(A^{t}(i, j)\right)^{2}=A^{2 t}(i, i)
$$

for all $i=0,1, \ldots,|G|-1$.

Proof: Let $\Gamma(G, \Omega)$ is bipartite. Then

$$
\begin{aligned}
|G| \cdot\left\|A^{t}(r, \cdot)-U(\cdot)\right\|^{2} & =\sum_{s=0}^{|G|-1}\left(A^{t}(r, s)-U(s)\right)^{2} \\
& =\sum_{s=0}^{|G|-1}\left(\left(A^{t}(r, s)\right)^{2}-\frac{2}{|G|} A^{t}(r, s)+\frac{1}{|G|^{2}}\right) \\
& =\sum_{s=0}^{|G|-1}\left(A^{t}(r, s)\right)^{2}-\frac{1}{|G|} \\
& =A^{2 t}(r, r)-\frac{1}{|G|}
\end{aligned}
$$


where the fact that $\sum_{s=0}^{|G|-1} A^{t}(r, s)=1$ was used. Now since $\Gamma(G, \Omega)$ is vertex transitive, $A^{t}(i, i)=A^{t}(j, j)$ for all $t$ and $i, j=0,1, \ldots,|G|-1$. Therefore,

$$
A^{2 t}(r, r)=\frac{1}{|G|} \sum_{r=0}^{|G|-1} A^{2 t}(r, r)=\frac{1}{|G|} \operatorname{tr}\left(A^{2 t}\right)=\frac{1}{|G|}\left(\lambda_{0}^{2 t}+\lambda_{1}^{2 t}+\cdots+\lambda_{|G|-1}^{2 t}\right),
$$

and since $\lambda_{0}=1$, this leaves

$$
A^{2 t}(r, r)-\frac{1}{|G|}=\frac{1}{|G|} \sum_{j=1}^{|G|-1} \lambda_{j}^{2 t}
$$

which completes the argument for the non-bipartite case. The bipartite case is obtained by repeating the same argument, but replacing $U$ with $W_{G_{i}}$, and $\lambda_{|G|-1}^{2 t}$ with 1 .

The Theorem of Section 6: The theorem is proved by gathering results dispersed throughout $[20]{ }^{32}$ As mentioned, the result is features prominently in the work of Diaconnis (Chapter 3 of [13] illustrates the use of the material in studying the convergence of Markov processes on groups. It also provides additional references.) The remainder of the section consists of a long build-up of material, followed by a short proof. The proof is simply a matter of fitting together the many pieces that have been introduced along the way.

Let $G$ be a finite group with identity $e$, and let $G L_{d}$ be the group of invertible $d \times d$ matrices over the complex numbers. As was mentioned in the text, a representation of $G$ is a homomorphism $\rho: G \rightarrow G L_{d}$. An equivalent concept, that will be more useful in the present context, is that of a $\mathbb{C} G$-module([20], Chapter 4). This consists group $G$ and a vector space $V$ over $\mathbb{C}$, together with a group action of $G$ on $V$ that respects the linear structure of $V$. Specifically, the action, $V \times G \rightarrow G$ must adhere to the conditions

1. $v g \in V$,

2. $v(g h)=(v g) h$,

3. $v e=v$,

\footnotetext{
${ }^{32}$ The machinery here is from the theory of representation of finite groups. The book by James and Liebeck, which is pitched at the level of an undergraduate student, is an excellent introduction to this material; it is clearly written and reasonably comprehensive. A more streamlined, yet still highly readable presentation is provided in the first chapter of the graduate-level text by Sargan, ([?]. The material is presented with a heavier emphasis on Fourier analysis on groups in the monograph by Diaconnis, [13].
} 
4. $(c v) g=c(v g)$,

5. $(u+v) g=u g+v g$,

where $u, v \in V, g, h \in G$, and $c \in \mathbb{C}$. Conditions (1) - (3) state that $G$ acts on $V$ by mean of right-multiplication, while conditions (4) and (5) require that the action respect the vector space structure. Every representation $\rho: G \rightarrow G L_{d}$ can be identified with a $\mathbb{C} G$ module, by specifying the action $v g=v \rho(g) .{ }^{33}$ The opposite is also true, since under the requirements (1) - (5), each $g \in G$ is a linear transformation on $V$, and thus, once a basis is chosen, can be represented as a matrix. Moreover this identification of each element of $G$ with a matrix is consistent with the group operation on $G$. The most important $\mathbb{C} G$-module is the group algebra, denoted by $\mathbb{C}[G]$. Here each element of $G$ is regarded as a basis vector, and the vector space is the set of all sums $a_{1} g_{1}+g_{2}+\ldots a_{n} g_{n}, a_{i} \in \mathbb{C}$ and $g_{i} \in G$, under the obvious rules for vector addition and scalar multiplication. ${ }^{34}$ The action of $G$ on $\mathbb{C}[G]$ is given by $\left(\sum_{x \in G} a_{x} x\right) g=\sum_{x \in G} a_{x}(x g)$. Note that this action can be extended linearly to all formal sums of the type $\sum_{y \in G} b_{y} y$ by requiring that

$$
\left(\sum_{x \in G} a_{x} x\right)\left(\sum_{y \in G} b_{y} y\right)=\sum_{x \in G} \sum_{y \in G} a_{x} b_{y}(x y)
$$

Once one gathers terms in the sum on the righthand side, one is left with $\sum_{g \in G} c_{g} g$, where $c_{g}=\sum_{x \in G} a_{x} b_{x^{-1} g}$, which is just the convolution product. In other words, the group action of $G$ on the vector space $\mathbb{C}[G]$ extends naturally to the operation of convolution. Under this operation, $\mathbb{C}[G]$ becomes an algebra (hence its name). This is also the important link between the economic model in this paper, and machinery of group representations.

A vector subspace $W$ of $V$ is a $\mathbb{C} G$-submodule of $V$ if $w g \in W$ for all $w \in W$ and $g \in G$. In other words, a subspace $W$ of $V$ is $\mathbb{C} G$-submodule if it is a $\mathbb{C} G$-module in its own right. A $\mathbb{C} G$-module is irreducible if it contains no $\mathbb{C} G$-submodule other than $\{0\}$ or $V$; otherwise, it is reducible. Here is the first major result (Theorem 10.5, p. 91 of $[20])[20]$ : The group algebra $\mathbb{C}[G]$ admits a direct sum decomposition, $\mathbb{C}[G]=$ $U_{1} \oplus U_{2} \oplus \cdots \oplus U_{n}$ where each $U_{i}$ is an irreducible $\mathbb{C} G$-submodule, and every irreducible $\mathbb{C} G$-module is isomorphic to one of the $U_{i}$ 's. Also, the number of copies of $U_{i}$ appearing

\footnotetext{
${ }^{33}$ Here the vector $v \in \mathbb{C}^{d}$ is right-multiplied by the matrix $\rho(g) \in G L_{d}$.

${ }^{34}$ Specifically, if $\sum_{g \in G} a_{g} g \in \mathbb{C}[G], \sum_{g \in G} b_{g} g \in \mathbb{C}[G]$, and $c \in \mathbb{C}$, then vector addition is defined according to $\sum_{g \in G} a_{g} g+\sum_{g \in G} b_{g} g=\sum_{g \in G}\left(a_{g}+b_{g}\right) g$, while scalar multiplication is given by $c\left(\sum_{g \in G} a_{g} g\right)=\sum_{g \in G}\left(c a_{g}\right) g$.
} 
in the direct sum $U_{1} \oplus \cdots \oplus U_{n}$ is equal to the dimension of $U_{i}$ (Theorem 11.9, page 100 of $[20])$.

Let $V$ be a $\mathbb{C} G$-module, and let $B$ be any basis for $V$. For any $x \in G,[x]_{B} \in G L_{d}$ denote the matrix corresponding to the linear transformation induced by the action $v \mapsto v x, v \in V$. The character of $V$ is the function $\chi: G \rightarrow \mathbb{C}$ defined according to $\chi(x)=\operatorname{tr}\left([x]_{B}\right)$ for all $x \in G$. The value of $\chi(x)$ is independent of the choice of basis. If $V$ is irreducible, then the corresponding character is said to be irreducible. As mentioned in Section 6, characters are class functions, and, more importantly, the irreducible characters comprise an orthonormal basis for the set of all class functions on G. (Corollary 15.4, page 153 and Theorem 14.12, page 140, [20].)

Here is a crucial fact (Proposition 9.18, page 84, [20]), although it does not play a direct role in the proof of the Theorem. Let $Z(\mathbb{C}[G])$ denote the center of the group algebra. (Formally $z \in Z(\mathbb{C}[G]$ ) if and only if $z x=x z$ for all $x \in \mathbb{C}[G]$ ). Let $V$ be an irreducible $\mathbb{C} G$-module, and let $z$ be any member of $Z(\mathbb{C}[G])$. Then there is a $\lambda \in \mathbb{C}$ such that $v z=\lambda v$ for all $v \in V$. The theorem of Section 6 is ultimately due to the fact that $\delta_{\Omega}$ is built from elements in $Z(\mathbb{C}[G])$.

Let $C_{1}, C_{2}, \ldots, C_{r}$ be the conjugacy classes in $G$ and for any $C_{i}$, let $\sigma_{i}$ denote the class sum $\sum_{x \in C_{i}} x$. Here is another significant result: The class sums $\sigma_{1}, \ldots, \sigma_{r}$ comprise a basis for $Z(\mathbb{C}[G])$ (Proposition 12.21, page 114). It now follows that if $V$ is an irreducible $\mathbb{C} G$-module, then there is a $\lambda_{i} \in \mathbb{C}$ such that $v \sigma_{i}=\lambda_{i} v$. Moreover, if $\chi$ is the character of $V$, then by taking traces of both sides of this equality (Lemma 22.7, page 247), one finds that $\lambda_{i}=\frac{\left|C_{i}\right|}{\chi(e)} \chi(x)$, where $x$ is any element in $C_{i}$. Given an arbitrary function $f: G \rightarrow \mathbb{C}$, let $\bar{f}$ denote the function where $\bar{f}(x)$ is the complex conjugate of $f(x)$. Another minor fact is that if $\chi: G \rightarrow \mathbb{C}$ is an irreducible character, then so is $\bar{\chi}: G \rightarrow \mathbb{C}$ (Proposition 13.15 , p. 126). In fact, if $\chi$ is the character for a $\mathbb{C} G$-module $V$, then $\bar{\chi}$ is the character for the dual of $V$ (that is, the set of linear functions on $V$ ). Also, if $\chi$ is irreducible, then so is $\bar{\chi}$ (Proposition 13.15, page 126).

Finally, let $V_{i}$ be an irreducible $\mathbb{C} G$-module of dimension $d_{i}$, and then let $W_{i}$ denote the direct sum of $d_{i}$ copies of $V_{i}$. As mentioned above, $\mathbb{C}[G]$ can be written as $\mathbb{C}[G]=$ $W_{1} \oplus \ldots \oplus W_{r}$. Therefore, the identity $1 \in \mathbb{C}[G]$ can be decomposed as the sum $1=$ $e_{1}+\ldots+e_{r}, e_{i} \in W_{i}{ }^{35}$ Since $e_{i} \in W_{i}$ it follows that $e_{j} \sigma_{i}=\lambda_{i} e_{j}$ for any $i, j \in\{1, \ldots, r\}$. However, more importantly, $e_{i}=\frac{\chi_{i}(e)}{|G|} \sum_{x \in G} \overline{\chi_{i}}(x)$, where $\chi_{i}$ is the irreducible character for $V_{i}$ (Proposition 14.10, page 138). In other words, $\overline{\chi_{i}} \in W_{i}$, so $\overline{\chi_{i}} \sigma_{j}=\lambda_{j} \overline{\chi_{i}}$ where $\lambda_{j}=\frac{\left|C_{j}\right|}{\chi_{i}(e)} \chi_{i}(x)$ for $x \in C_{j}$.

In order to prove the theorem, it only remains to translate facts about elements of

\footnotetext{
${ }^{35}$ Although it is not required for the Theorem, the $e_{i}$ 's have the remarkable property of being projections: $w e_{i}=w$ if $w \in W_{i}, w e_{i}=0$ if $w \notin W_{i}$, and $e_{i}^{2}=e_{i}$ (pages 137-138).
} 
$\mathbb{C}^{|G|}$ into facts about elements in $\mathbb{C}[G]$. Any function $f \in \mathbb{C}^{|G|}$ can be identified with the element $\sum_{g \in G} f(g) g$ in $\mathbb{C}[G]$. Under this identification, the operation $f * \delta_{\Omega}$ defined in Section 2 (according to which $m_{t} * \delta_{\Omega}=m_{t+1}$ ) for any $f$, is equivalent to right multiplication of $f$ by $\delta_{\Omega}$ in $\mathbb{C}[G]$, as described by $(24)$.

The theorem of Section 6 now follows. Let $\Gamma(G, \Omega)$ be a Cayley graph whose generating set adheres to Assumptions $G 0-G 4$. In particular, let $\Omega=C_{1} \cup \cdots \cup C_{s}$. As an element of $\mathbb{C}[G], \delta_{\Omega}$ is just $\frac{1}{|\Omega|} \sum_{i=1}^{s} \sigma_{i}$, so that if $\chi_{i}$ is any irreducible character for the $\mathbb{C} G$-module then $\chi * \delta_{\Omega}$ is

$$
\begin{aligned}
\chi_{i} \cdot \delta_{\Omega} & =\chi_{i}\left(\frac{1}{|\Omega|}\left(\sigma_{1}+\cdots+\sigma_{s}\right)\right) \\
& =\frac{1}{|\Omega|}\left(\chi_{i} \sigma_{1}+\cdots+\chi_{i} \sigma_{s}\right) \\
& =\frac{1}{|\Omega|}\left(\frac{\left|C_{1}\right|}{\chi_{i}(e)} \overline{\chi_{i}}\left(x_{1}\right)+\cdots+\frac{\left|C_{s}\right|}{\chi_{i}(e)} \overline{\chi_{i}}\left(x_{s}\right)\right) \chi_{i}
\end{aligned}
$$

where $x_{j} \in C_{j}$. (Note that $\chi_{i}(e)$ is real, so it replaces $\overline{\chi_{i}(e)}$ in the preceeding expression.) Finally, under Assumption $G 2$, if $x$ belongs to one of the conjugacy classes $C_{1}, \ldots C_{s}$, then so must $x^{-1}$; therefore if $\left|C_{j}\right| \overline{\chi_{i}\left(x_{j}\right)}$ appears in the sum above, then so must $\left|C_{j}\right| \chi_{i}\left(x_{j}\right)$. Consequently, $\frac{1}{|\Omega| \chi_{i}(e)}\left(\left|C_{1}\right| \overline{\chi_{i}}\left(x_{1}\right)+\cdots+\left|C_{s}\right| \overline{\chi_{i}}\left(x_{s}\right)\right)$ is equal to its complex conjugate, and is therefore real.

\section{References}

[1] Aldous, David, and James Fill, Reversible Markov Chains and Random Walks on Graphs at http://www.stat.berkeley.edu/ aldous/RWG/book.html

[2] Artin, Michael, Algebra, Prentice-Hall Inc., Englewood Cliffs, New Jersey, 1991.

[3] Beineke, Lowell, and Robin Wilson, Eds., Topics in Algebraic Graph Theory, Cambridge University Press, Cambridge, 2004.

[4] Berentsen, Aleksander, Gabriele Camera, and Christopher Waller, "The Distribution of Money Balances and the Non-Nuetrality of Money," International Economic Review, 46, (2005) pages 465-87.

[5] Bernanke, Ben, The Great Moderation, speech delivered at the meetings of Eastern economic Association, Februaury 20, 2004, http://www.federalreserve.gov/boarddocs/speeches/2004/20040220/default.htm 
[6] Bhattacharya, Joiydeep, Joseph Haslag, and Antoine Martin, "Heterogeneity, Redistribution, and the Friedman Rule," International Economic Review, 46, (2005) pages 437-54.

[7] Biggs, Norman, Algebraic Graph Theory, Second Edition, Cambridge Mathematical Library, Cambridge University Press, Cambridge, 1993.

[8] Blanchard, Olivier, and John Simon, "The Long and Large Decline in U.S. Output Volatility," Brookings Papers on Economic Activity, 1, (2001), pages 135-164.

[9] Bremaud, Pierre, Markov Chains: Gibbs Fields, Monte Carlo Simulation, and Queues, Springer-Verlag, New York, 1999.

[10] Chung, Fan R.K., Spectral Graph Theory, Regional Conference Series in Mathematics, No. 92, American Mathematical Society, Providence, R.I, 1997.

[11] Christiano, Lawrence, and Martin Eichenbaum, "Liquidity Effects and the Monetary Transmission Mechanism," American Economic Review, 82, (1992) pages 346-53.

[12] Chung, Fan R. K., Spectral Graph Theory, Regional Conference Series in Mathematics, No. 92, American Mathematical Society, 1997.

[13] Diaconis, Persi, Group Representations in Probability and Statistics, Institute of Mathematical Statistics, Hayward California, 1988.

[14] Diaconis, Persi, "The cutoff phenomenon in Finite Markov chains," Proceedings of the National Academy of Sciences, USA, Vol. 93, pages 1659 - 1664, February, 1996.

[16] Fuerst, Timothy, "Liquidity, Loanable Funds, and Real Economic Activity," Journal of Monetary Economics, 29, (1992) pages 3-24.

[17] Godsil, C. and R. Royle, Algebraic Graph Theory, Springer-Verlag, New York, 2001.

[18] Grossman, Sanford, and Laurence Weiss, "A Transactions-Based Model of the Monetary Transmission Mechanism," American Economic Review, 73, (1983) pages 871880.

[19] Herstein, I.N., Topics in Algebra, 2nd Ed., John Wiley and Sons, 1975.

[20] James, Gordon, and Martin Liebeck, Representations and Characters of Groups, Second Edition, Cambridge University Press, Cambridge, 2001. 
[21] Korner, T.W., Fourier Analysis, Cambridge University Press, 1988.

[22] Lucas, Robert E., Jr., "Equilibrium in a Pure Currency Economy," Economic Inquiry, 18, (1980) pages 203-220.

[23] Lucas, Robert E., Jr., "Liquidity and Interest Rates," Journal of Economic Theory, 50, (1990) pages 237-264.

[24] Saloffe-Coste, Laurent, Random Walks on Finite Groups, in Probability on Discrete Structures, Harry Ketsen Ed., Encyclopaedia of Mathematical Sciences, Vol. 110, Springer, Berlin, 2004.

[25] McConnell, Margarte, and Gabriel Perez-Quiros, "Output Fluctuations in the United States: What has Changed Since the Early 1980's?" American Economic Review, 90, (2000), pages 1464-1476.

[26] Sargan, Bruce E., The Symmetric Group: Representations, Combinatorial Algorithms, and Symmetric Functions, Second Edition, Springer-Verlag, New York, 2000.

[27] Stock, James, and Mark Watson, "Has the Business Cycle Changed: Evidence and explanations," prepared for the Federal Reserve Bank of Kansas City symposium "Monetary Policy Under Uncertainty," Jackson Hole, Wyoming, August 28-30, 2003.

[28] "Models of Money with Spatially Separated Agents," in Models of Monetary Economies, John Kareken and Neil Wallace, eds., Federal Reserve Bank of Minneapolis, 1980, pages 265-303. 